\title{
Probing the dynamical and X-ray mass proxies of the cluster of galaxies Abell S1101*
}

\author{
Andreas Rabitz ${ }^{1}$, Yu-Ying Zhang ${ }^{2}$, Axel Schwope ${ }^{1}$, Miguel Verdugo ${ }^{3}$, Thomas H. Reiprich ${ }^{2}$, and Matthias Klein ${ }^{4}$ \\ ${ }^{1}$ Leibniz-Institut für Astrophysik Potsdam (AIP), An der Sternwarte 16, 14482 Potsdam, Germany \\ e-mail: arabitz@aip.de \\ 2 Argelander-Institut für Astronomie, Auf dem Hügel 71, 53121 Bonn, Germany \\ 3 Department for Astrophysics University of Vienna, Türkenschanzstr. 17, 1180 Vienna, Austria \\ ${ }^{4}$ Max-Planck-Institut für extraterrestrische Physik, Giessenbachstr. 1, 85748 Garching, Germany
}

Received 2 June 2016 / Accepted 23 August 2016

\begin{abstract}
Context. The galaxy cluster Abell S1101 (S1101 hereafter) deviates significantly from the X-ray luminosity versus velocity dispersion relation $(L-\sigma)$ of galaxy clusters in our previous study. Given reliable X-ray luminosity measurement combining XMM-Newton and ROSAT, this could most likely be caused by the bias in the velocity dispersion due to interlopers and low member statistic in the previous sample of member galaxies, which was solely based on 20 galaxy redshifts drawn from the literature.

Aims. We intend to increase the galaxy member statistics to perform precision measurements of the velocity dispersion and dynamical mass of S1101. We aim for a detailed substructure and dynamical state characterization of this cluster, and a comparison of mass estimates derived from (i) the velocity dispersion $\left(M_{\text {vir }}\right)$, (ii) the caustic mass computation $\left(M_{\text {caustic }}\right)$, and (iii) mass proxies from X-ray observations and the Sunyaev-Zel'dovich (SZ) effect.

Methods. We carried out new optical spectroscopic observations of the galaxies in this cluster field with VIMOS, obtaining a sample of $\sim 60$ member galaxies for S1101. We revised the cluster redshift and velocity dispersion measurements based on this sample and also applied the Dressler-Shectman substructure test.

Results. The completeness of cluster members within $r_{200}$ was significantly improved for this cluster. Tests for dynamical substructure do not show evidence of major disturbances or merging activities in S1101. We find good agreement between the dynamical cluster mass measurements and X-ray mass estimates, which confirms the relaxed state of the cluster displayed in the 2D substructure test. The SZ mass proxy is slightly higher than the other estimates. The updated measurement of $\sigma$ erased the deviation of S1101 in the $L-\sigma$ relation. We also noticed a background structure in the cluster field of S1101. This structure is a galaxy group that is very close to the cluster S1101 in projection but at almost twice its redshift. However the mass of this structure is too low to significantly bias the observed bolometric X-ray luminosity of S1101. Hence, we can conclude that the deviation of S1101 in the $L-\sigma$ relation in our previous study can be explained by low member statistics and galaxy interlopers, which are known to introduce biases in the estimated velocity dispersion.
\end{abstract}

Key words. galaxies: clusters: individual: Abell S1101 - cosmology: observations - X-rays: galaxies: clusters galaxies: clusters: intracluster medium - galaxies: kinematics and dynamics - methods: data analysis

\section{Introduction}

Galaxy clusters memorize structure formation (for instance Borgani \& Guzzo 2001), and cluster surveys have thus been widely used to constrain the cosmological parameters such as the dark energy content (e.g. Mantz et al. 2014). Various methods such as optical (see also Gladders \& Yee 2005), X-rays (for example Böhringer et al. 2001), weak lensing (e.g. Schneider 1996), and the Sunyaev-Zel'dovich (SZ; Sunyaev \& Zeldovich 1972) effect (compare for instance Vale \& White 2006), could potentially lead to biases in determining the mass function in the cluster cosmological experiments. X-ray selects systems containing hot gas, as a sign of virialization (for instance Weinberg \& Kamionkowski 2002), and are considered to provide a cleaner and more complete selection regarding mass.

To improve our knowledge of the X-ray selection method, we investigated the $\mathrm{X}$-ray bolometric luminosity versus velocity

* We have made use of VLT/VIMOS observations taken with the ESO Telescope at the Paranal Observatory under programme 087.A-0096. dispersion $\left(L_{\mathrm{bol}}-\sigma\right)$ relation of 62 clusters in an X-ray fluxlimited sample of 64 clusters (Zhang et al. 2011). The observed scatter in the relation in our observational data was thought to be mainly related to the presence of cool cores but was even present after the cool-core correction in deriving the X-ray luminosity. To study the other possible origin of this scatter, in Zhang et al. (2011) we also compared the $L_{\mathrm{bol}}-\sigma$ relations between our observational sample and a sample of 21 clusters and groups from very high-resolution simulations with and without active galactic nuclei (AGN) feedback provided by Puchwein et al. (2008). The simulated sample with AGN feedback matches well with the observational sample of 56 clusters, excluding six clusters with less than 45 cluster galaxy redshifts $\left(n_{\text {gal }}<45\right)$, in which AGN feedback in different phases explains the increasing scatter of our observational sample towards the low-mass end. The velocity dispersion estimates for those $n_{\mathrm{gal}}<45$ clusters are about a factor of two lower than the $L_{\mathrm{bol}}-\sigma$ predictions such that they cause more than two times larger scatter than that of the remaining clusters. This indicates that AGN feedback cannot be the main origin of the scatter of the $L_{\mathrm{bol}}-\sigma$ relation for those $n_{\mathrm{gal}}<45$ systems. 
We suspect that systematic uncertainties in the velocity dispersion estimates (e.g. Biviano et al. 2006; Saro et al. 2013) may play a stronger role than the cluster physics, such as AGN feedback, in causing the scatter. We confirmed our guess by carefully testing systematic uncertainties using the simulated sample due to interlopers and the selection of cluster members regarding the aperture radius and mass limit in Zhang et al. (2011). The systematic uncertainties due to those effects are up to $40 \%$; the low member statistics in recovering the caustic amplitude may play an important role in causing the bias (also see Clerc et al., in prep).

As a second-order effect, we found in Zhang et al. (2011) that interlopers always bias the velocity dispersion measurements towards lower values for our simulated sample except for one cluster. There are significant numbers of galaxies within 1.2 Abell radii that are not in the virialized regions for poor systems in our simulated sample. As also shown in Fig. 10 in Biviano et al. (2006), unrecognized interlopers that are outside the virial radius $\left(r_{\mathrm{vir}}\right)$ but dynamically linked to the host cluster, and that do not form major substructures, bias the $\sigma$ estimate towards lower values than cluster galaxies. There are also similar studies based on other simulations (e.g. Saro et al. 2013). Particularly, the line-of-sight velocity of the galaxies with available spectroscopic redshifts (de Vaucouleurs et al. 1991; Shectman et al. 1996; Zabludoff \& Mulchaey 1998; Jones et al. 2009) as a function of the projected radius shows a box-shape instead of a caustic shape for one of the $n_{\text {gal }}<45$ clusters, that is Abell S1101 (S1101 hereafter). This indicates that the chance of having a high percentage of unrecognized interlopers at large radii is likely high for this cluster. To disentangle the scatter driven by interlopers and by other physics in the $L_{\mathrm{bol}}-\sigma$ relation and to constrain possible bias in the X-ray selection method, we carried out a detailed study on S1101 based on newly awarded VLT/VIMOS (Very Large Telescope/VIsible Multi-Object Spectrograph; Le Fèvre et al. 2003) spectroscopic observations together with XMM-Newton and ROSAT X-ray data. We note that S1101 was detected as RXC J2313.9-4243 in Böhringer et al. (2004) in the ROSAT All-Sky Survey (RASS; Trümper 1992). It is also listed as SPT-CL J2313-4243 detected through the SZ effect in Bleem et al. (2015).

Throughout the paper we assume a standard cosmology with $\Omega_{\mathrm{M}}=0.3, \Omega_{\Lambda}=0.7$ and $H_{0}=70 \mathrm{~km} \mathrm{~s}^{-1} \mathrm{Mpc}^{-1}$. Thus, with an angular diameter distance of $D_{\mathrm{A}}=224 \mathrm{Mpc}$, a scale of $1^{\prime}$ corresponds to an extent of $\sim 65 \mathrm{kpc}$ at the cluster redshift $z_{\mathrm{cl}}=$ $0.05601 \pm 0.00027$. Errors are given as $95 \%$ confidence intervals, unless stated otherwise.

\section{Optical imaging and target selection}

The cluster S1101 was observed at the ESO/MPG $2.2 \mathrm{~m}$ telescope with the wide-field imager (WFI; Baade et al. 1999) in the $V / 89-, R_{\mathrm{c}} / 162$, and $I_{\mathrm{c}}$ bands under non-photometric conditions (PI: Reiprich) as a bad weather backup target. We reduced the data using the THELI pipeline (Erben et al. 2005; Schirmer 2013). The co-added images have $1^{\prime \prime} .5$ seeing in $V / 89$ and $R_{\mathrm{c}} / 162$, and 3'. 1 seeing in $I_{\mathrm{c}}$. The relatively poor quality of the imaging combined with sub-optimal weather makes the determination of the zero point not better than $0.15 \mathrm{mag}$.

The combination of those filters is not the best to bracket the $4000 \AA$ break to isolate fully the red sequence for the colour selection of the candidate cluster galaxies at the redshift of S1101, but this combination was also chosen to select active galaxies. Furthermore, a precise preselection of cluster member galaxies according to their colours was hindered by the bad seeing and resulting improper photometric calibration. The existing 2MASS (Skrutskie et al. 2006) data also failed to improve the photometric calibration. Most 2MASS sources are also saturated for the galaxies in our observations. The standard fields are not of sufficient quality to calibrate the $B$-band FORS data.

Still, from the reduced imaging data we are able to extract catalogues using SExtractor (Bertin \& Arnouts 1996) in dual-image mode with the $R_{\mathrm{c}} / 162$ band as detection image. We filtered the catalogue for objects where the CLASS_STAR of SExtractor indicates a likely galaxy and the fluxes in the respective bands are less than those of the apparent bright central galaxy (BCG).

Our VIMOS spectroscopic follow-up actually covers a larger sky area than the WFI field. Therefore, we have to rely on the VIMOS pre-imaging, performed with the $R$ filter, to select objects that appear as bright ellipticals for the incremental region. We also reduced the pre-imaging of our VIMOS campaign with the THELI pipeline. The photometry is calibrated against the $\mathrm{Su}-$ perCOSMOS catalogues in the Vega system. The offset between SuperCOSMOS and the calibrated VIMOS $R$-band catalogue is about 0.3 mag, which is rather significant. It may be caused by calibrations in either of the observations and/or filter differences. Nevertheless, we are able to provide uniform photometry for all VIMOS spectroscopic sources thanks to the calibrated VIMOS pre-imaging.

Follow-up priorities were assigned according to their luminosities to maximize the signal-to-noise ratio ( $\mathrm{S} / \mathrm{N}$ herafter) and the effective survey area within the given observation time and positional constraints set by multi-object spectrographs. We present the sky positions of the objects in our survey field in the left panel of Fig. 1. Furthermore, the right panel of Fig. 1 shows a colour-colour diagram of the galaxies in the WFI field, which we used to selected the candidates for the VIMOS spectroscopic follow-up. Our aim to cover all available space on the slit masks led to the selection of additional sources that appeared as bright ellipticals in cases where colour-selected sources could not be targeted as a result of typical technical restrictions of multiobject spectrographs. Qwing to bad weather, the spectroscopic follow-up shows an artificial elongation along the southeastnorthwest direction as not all scheduled VIMOS pointings in the cluster field were performed.

\section{Optical spectroscopic data analysis and results}

\subsection{Spectroscopic data and data reduction}

The spectroscopic follow-up of potential member galaxies of S1101 was conducted under ESO proposal ID 087.A-0096 (PI: Zhang). We applied for the wide-field and survey-probed capabilities of VIMOS at the UT3 (Melipal) of the ESO VLT, and were granted $22.5 \mathrm{~h}$ in total for S1101 and A2597, in which only the observations of S1101 were almost completed for $\sim 11.5 \mathrm{~h}$. The field of view of VIMOS is split into quadrants of one single CCD each, $4 \times 7^{\prime} \times 8^{\prime}$ with small gaps between the quadrants. Using spectroscopic mode, multi-object slit masks were manufactured for seven pointings that cover the cluster field of S1101 out to the virial radius. All spectroscopic data for this programme were taken between June and September 2011. Typical science exposure times were $1150 \mathrm{~s}$ and between three and nine individual exposures per pointing were taken. We used the HR-Blue grism of VIMOS in order to achieve a redshift accuracy high enough to identify cluster substructures and obtain a reliable $\sigma$ estimate and its deviation from the Gaussian distribution (e.g. Maurogordato et al. 2008). We measured the full width 

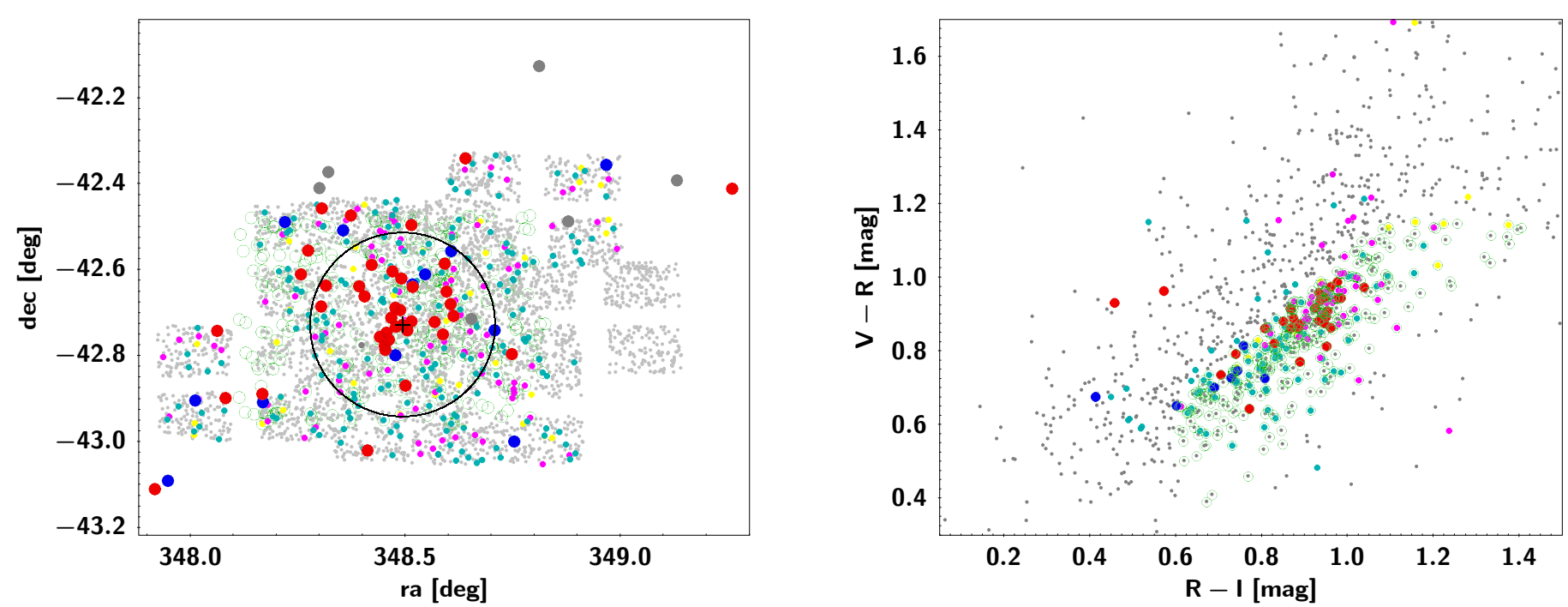

Fig. 1. Skypositions (left panel) and WFI colour-colour diagram (right panel) of the objects in the cluster field of S1101. Small grey dots show all sources detected from the VIMOS pre-imaging and the WFI imaging for the left and the right panel, respectively. Green circles indicate WFI colour-colour selected candidates for the spectroscopic follow-up. The imaging candidates that were spectroscopically observed but classified as non-members are indicated with magenta and cyan dots for galaxies with passive and active spectral types and yellow dots for stars. Large dots in red, blue, and grey correspond to spectroscopically verified cluster members with passive, active, and unknown spectral types. The black cross denotes the cluster X-ray centre. The big black circle shows the area within a projected distance of $r_{500}$. Because of bad weather, the spectroscopic follow-up shows an artificial elongation along the southeast-northwest direction as not all scheduled VIMOS pointings in the cluster field were performed.

at half maximum (FWHM) of unblended emission lines in arclamp exposures, taken with the same mask and hence slit width as the science frames. This information allowed inferences on the spectral resolution, $R \equiv \lambda \cdot \Delta \lambda^{-1}$. Our chosen set-up falls within $1400 \lesssim R \lesssim 2070$, considering the instrumental wavelength range of 4200-6200 $\AA$ and central 1 " slits. The quality of the reduced spectra allows us to not only detect emission lines, but because of their high $\mathrm{S} / \mathrm{N}$ we can also detect prominent absoption features of early-type galaxies, for example, CaII

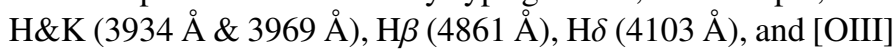
(4959 ̊ and $5007 \AA$ ).

The data were reduced within ESO-Midas (Banse et al. 1983 ) with a suitably tailored set of scripts bundled together for a largely automatic pipeline reduction. The reduction workflow includes the following tasks: de-biasing of raw input frames, flat-fielding and cosmic filtering of scientific frames, wavelength calibration, weighted extraction of one-dimensional (1D) spectra (following Horne 1986) including the generation of respective error spectra, the creation of two-dimensional (2D) sky-subtracted frames and, finally, the co-addition of single fluxcalibrated spectra.

We were able to extract a total of 492 spectra, of which 457 were of sufficient quality for our scientific use. We initially examined all spectra using EZ (Garilli et al. 2010) to get the initial measure of the quality and redshift of each reduced spectrum. For spectra within a generous range around the cluster redshift $(0.04 \leq z \leq 0.08)$ the final redshift was computed as the average of independent Gaussian fits to available spectral features in the individual spectra to sustain high accuracy for tentative cluster members. The CaII H\&K (3934 ̊ \& $3969 \AA$ A) absorption lines for passive galaxies were fitted primarily. In addition we used the $G$ band (4304 $\AA$ ), $\mathrm{H} \delta$ (4103 $\AA$ ), $\mathrm{H} \gamma(4340 \AA), \mathrm{H} \beta$ (4861 $)$ ), and MgII (5175 $\AA$ ) absorption features, as well as

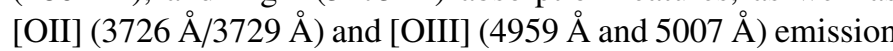
lines, when permitted by the spectral range and feature quality.
We chose to correct our individual redshifts with respect to the barycentre of the solar system to reduce the systematics when averaging multiple observed sources and combining with galaxy samples from the literature, as described in the next subsection. By comparing acquisition images of slit masks with VIMOS pre-imaging data, we were able to allocate individual redshifted spectra to object coordinates from the Guide Star Catalogue-II. We take the unweighted average of the measured redshifts for objects observed multiple times.

The accuracy of our redshift determination is affected by the accuracy of the wavelength calibration, the precision with which emission and absorption lines can be determined and by the scatter of repeated redshift measurement for a given galaxy. These were added quadratically and result in a typical redshift error of $\Delta z \sim 0.00007\left(\sim 20 \mathrm{~km} \mathrm{~s}^{-1}\right)$, a value also found by Maurogordato et al. (2008) using the same spectroscopic set-up.

The VIMOS spectroscopic sample comprises 392 individual objects. According to the spectral classification, there are 220 active galaxies (with a clear indication of emission lines), 129 passive galaxies, 42 most likely late-type stars, and one Lyman- $\alpha$ emitter at redshift $\sim 3.4$. A redshift histogram of their redshift distribution, omitting the Lyman- $\alpha$ emitter, is given in Fig. 2.

\subsection{Incorporating public redshifts to our data}

We queried the NASA/IPAC Extragalactic Database (NED) and merged the 20 redshifts in the literature (i.e. de Vaucouleurs et al. 1991; Shectman et al. 1996; Zabludoff \& Mulchaey 1998; Jones et al. 2009) from objects at the tentative cluster redshift with our sample. We observed a scatter in redshift between galaxies from the literature and re-observations from this study but no systematics for the individual publications were found. Since not all sources in the literature have published quality assessments of their spectra, we use our redshifts instead of their NED counterparts, 


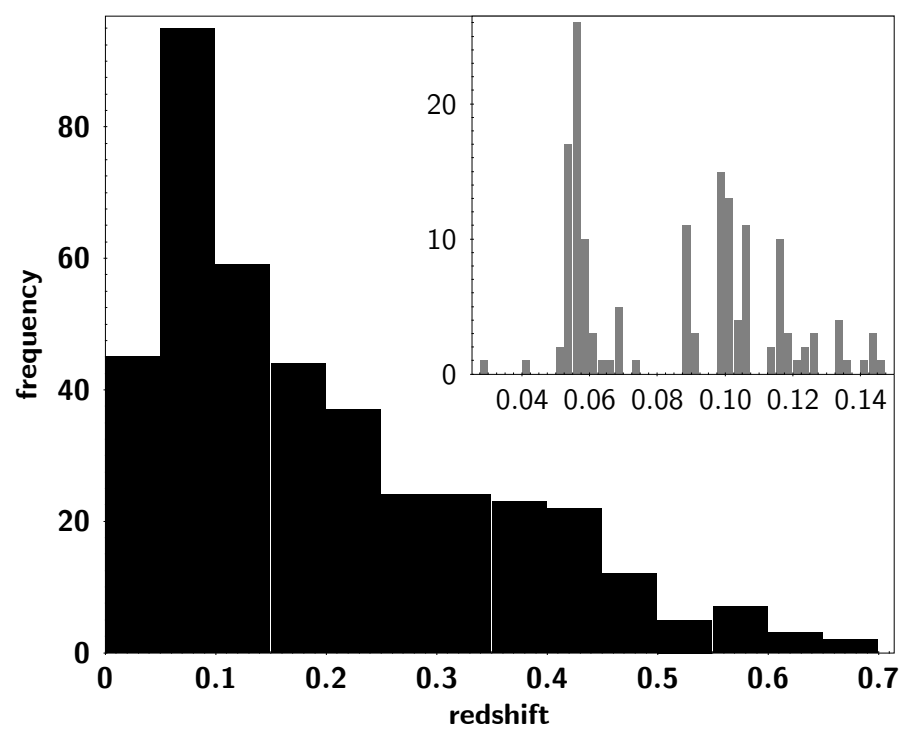

Fig. 2. Redshift histogram of the VIMOS spectroscopic sample excluding the Lyman- $\alpha$ emitter at $z \sim 3.4$. The inset in the upper right allows a closer inspection of a redshift window around S1101 at $z \sim 0.056$ Here, a large number of field galaxies as well as a possible background structure at $z \sim 0.1$ in our sample become recognizable.

and literature redshifts complementary to our work (e.g. outside the field covered by the VIMOS spectroscopy) were included.

Within the range of $0.05 \leq z \leq 0.065$ our redshift catalogue comprises 61 distinct galaxies (including 11 sources from the NED). We describe the cluster member selection in the following subsection. Additionally, a rather large number of background galaxies in the pool of our spectra at redshifts of around twice the cluster redshift (see Fig. 2) were detected. This apparent overdensity of galaxies at $z \sim 0.1$ is discussed further in Sect. 6.

\subsection{Member selection from spectroscopic data}

In order to investigate the dynamics of the cluster S1101, we need to assign cluster membership to the galaxies in our redshift catalogue. This is not trivial considering the fact that infalling or merging structures exist even in relaxed systems (see e.g. Zhang et al. 2012). We initially selected those galaxies fulfilling $\left|c z_{\mathrm{i}}-c \bar{z}\right| \leq 4000 \mathrm{~km} \mathrm{~s}^{-1}$ as cluster member candidates. Here, $c z_{\mathrm{i}}$ is the recessional velocity of the individual galaxy, and $c \bar{z}$ the mean velocity of the galaxy cluster $S 1101$ based on its redshift in the REFLEX Cluster Survey catalogue (Böhringer et al 2004), $\bar{z}=0.0564$.

A revised cluster redshift $\left(z_{\mathrm{cl}}\right)$ and velocity dispersion $\left(\sigma_{\mathrm{cl}}\right)$ was computed using the biweight estimates of location and scale following Beers et al. (1990). Following Biviano \& Girardi (2003), we applied the $\left|z_{\mathrm{cl}}-z_{\mathrm{i}}\right| \cdot c \leq 3 \sigma_{\mathrm{cl}}$ clipping to finalize the selection of member galaxies and repeated the computation. The resulting sample consists of 58 member galaxies, as visualized in Fig. 3.

A different approach of assigning cluster membership to galaxies is the method of "fixed gaps". Following the procedure by Katgert et al. (1996), we used a gap size of $1000 \mathrm{~km} \mathrm{~s}^{-1}$ and identified 59 galaxies as likely cluster members: the same 58 from above plus one additional at $z \sim 0.0635$ (see Fig. 3). Since the differences in velocity dispersion $(+5 \%)$ and mean cluster redshift $(+0.02 \%)$ are negligible, we stick to the previous procedure with iterative clipping based on the $\sigma_{\mathrm{cl}}$ for the following

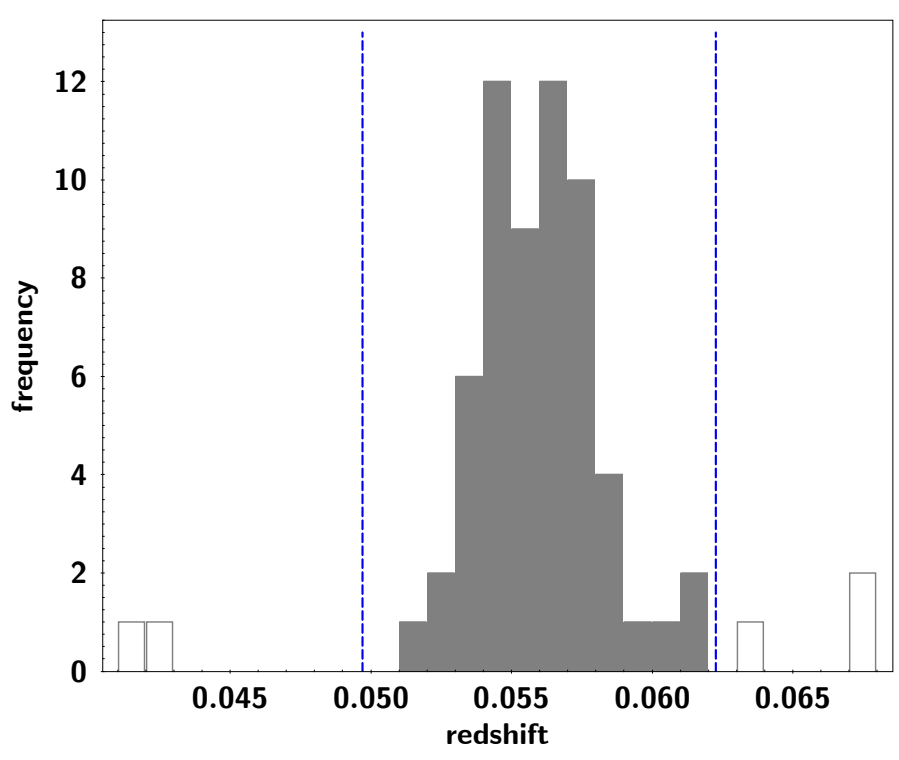

Fig. 3. Redshift distribution of initially selected member galaxies in the galaxy cluster $S 1101$. All visible galaxies were initially selected by $\mid c z_{\mathrm{i}}-$ $c \bar{z} \mid \leq 4000 \mathrm{~km} \mathrm{~s}^{-1}$ as described in Sect. 3.3. Estimators of location and scale $\left(z_{\mathrm{cl}}\right.$ and $\sigma$, respectively; see Beers et al. 1990) were used to clip interlopers. The resulting cluster galaxy candidates are indicated by the shaded histogram, while the blue vertical lines refer to the $\pm 3 \sigma$ interval of $\sigma_{\mathrm{cl}}$ around $z_{\mathrm{cl}}$ used for the clipping.

considerations. The equatorial coordinates and redshifts for all spectroscopic member galaxies identified in this work are given in Table B.1.

Figure 1 shows the sky positions of all spectroscopic member galaxies identified, together with their types being either passive or star-forming according to their characteristic spectral features (absorption- or emission-line dominated spectrum) once available. We note that some literature sources have no publicly available spectra for the classification. The restriction to this simple classification was chosen to gather information on the distribution of star-forming and passively evolving galaxies with respect to the cluster centric radii and a possible resulting bias for the $\sigma$ measurement. There is an obvious overdensity of passively evolving galaxies in our sample because we targeted the galaxy candidates selected by the photometric colour around the red sequence in our observations.

The final values of the cluster redshift and its velocity dispersion following the procedure of Beers et al. (1990) are $z_{\mathrm{cl}}=$ $0.05601 \pm 0.00027$ and $\sigma_{\mathrm{cl}}=\left(574_{-36}^{+38}\right) \mathrm{km} \mathrm{s}^{-1}$, respectively, are summarized in Table 2 with additional characteristic values of the cluster. Unfortunately the fraction of active galaxies is too low to compute an accurate $\sigma$ to directly compare $\sigma_{\text {pas }}$ and $\sigma_{\text {act }}$. The value measured for the passive fraction, however, agrees well within the errors with the total sample of cluster members (see Table 2).

The cluster radius $r_{\Delta}$ (e.g. $r_{500}$ ) was defined as the radius within which the mass density is $\Delta$ (e.g. 500) times of the critical density at the cluster redshift, $\rho_{\mathrm{c}}(z)=3 H_{0}^{2}(8 \pi G)^{-1} E^{2}(z)$, in which $E^{2}(z)=\Omega_{M}(1+z)^{3}+\Omega_{\Lambda}+\left(1-\Omega_{M}-\Omega_{\Lambda}\right)(1+z)^{2}$. The cluster mass can be estimated by the scaling relations calibrated by simulations from the velocity dispersion. An isothermal spherical mass model generally does not fit well to the mass distribution of clusters. The scaling relation based on such a model (e.g. Carlberg et al. 1997) thus provides a biased estimate. Instead, a NFW model (Navarro et al. 1997) is a better 
description of the halo mass profile of clusters. Based on the latter model, Munari et al. (2013) analysed different simulations of group- and cluster-sized structures involving various physical models. Therein they estimated the velocity dispersion versus cluster mass relation of the following tracers of the cluster mass distribution: (i) dark matter particles, (ii) subhalos and (iii) galaxies,

$$
\frac{\sigma_{1 \mathrm{D}}}{\mathrm{km} \mathrm{s}^{-1}}=A_{1 \mathrm{D}}\left[\frac{h(z) M_{200}}{10^{15} M_{\odot}}\right]^{\alpha}
$$

where $\sigma_{1 \mathrm{D}}$ is the line-of-sight velocity dispersion and $h(z)$ the Hubble parameter at the redshift $z$ normalized by $100 \mathrm{~km} \mathrm{~s}^{-1} \mathrm{Mpc}^{-1}$. Munari et al. (2013) conclude that the bestfit values of $A_{1 \mathrm{D}}$ and $\alpha$ using dark matter particles as the tracer are in general agreement with the NFW mass profile and provide consistent results as that from Evrard et al. (2008). The fits for subhalos and galaxies as tracers, on the other hand, show a steeper $\sigma-\mathrm{M}$ relation. Since we aim to determine the cluster mass of S1101 from the velocity dispersion of member galaxies, we used the full range of parameters found by tracing galaxies for the various physical models (ceased star formation and AGN feedback; compare Table 1 in Munari et al 2013), $A_{1 \mathrm{D}}=1169.75 \pm 11.45$, and $\alpha=0.3593 \pm 0.0068$. The corresponding mass and radius for the cluster S1101 are $M_{200}=1.92_{-0.42}^{+0.52} \times 10^{14} M_{\odot}$ and $r_{200}=1.169_{-0.093}^{+0.097} \mathrm{Mpc}$, given the uncertainies in $A_{1 \mathrm{D}}, \alpha$ and the error of our velocity dispersion $\sigma_{\mathrm{cl}}$ (see also Table 2). The bias in using the galaxies as the tracer is low for low- $z$ and low-mass clusters (Fig. 7 in Munari et al. 2013), which is the case of S1101.

\subsection{Two-dimensional kinematic structure}

The velocities of member galaxies scatter around the Hubble flow velocity of the underlying dark matter halo. According to the hierarchical clustering model, clusters grow through the accretion of galaxies and groups of galaxies falling into the cluster potential well. Knowledge of the substructures of a galaxy cluster is however crucial for the measurement of the line-ofsight velocity dispersion as well as its resulting mass proxy as substructures introduce biases in its velocity dispersion estimate (e.g. Biviano et al. 2006). The spatial and line-of-sight velocity distributions of galaxies allow us to distinguish member galaxies taking part in an idealized Gaussian velocity distribution around the cluster mean velocity from the infalling galaxies, groups of galaxies or even larger merging events.

Pinkney et al. (1996) extensively probed various approaches of substructure tests based solely on velocities, positions, or a combination of both. Accordingly, an advanced and robust test for substructure was described by Dressler \& Shectman (1988). They provided a statistical approach to test the 2D kinematic structure of a cluster using its galaxy positions and velocities as follows:

$\delta_{i}^{2}=\frac{N_{\mathrm{loc}}+1}{\sigma^{2}}\left[\left(\bar{v}_{i, \mathrm{loc}}-\bar{v}\right)^{2}+\left(\sigma_{i, \mathrm{loc}}-\sigma\right)^{2}\right]$.

Equation (2) makes use of $\bar{v}_{i, \text { loc }}, \bar{\sigma}_{i, \text { loc }}$, and $N_{\text {loc }}$ being the mean velocity, mean velocity dispersion, and galaxy number of the local group. Here $\bar{v}$ and $\sigma$ are the global mean velocity and the velocity dispersion of the cluster. The index $i$ runs over all galaxies in the sample. The reduced statistics for this test is given by

$\Delta_{\mathrm{DS}}=\sum_{i} \delta_{i}$
Table 1. Results of the Dressler-Shectman test applied to the galaxy cluster S1101.

\begin{tabular}{ccc}
\hline \hline$N_{\text {loc }}$ & $\Delta_{\text {DS }}$ & $P$ \\
\hline 5 & 30.781 & 0.882 \\
6 & 32.986 & 0.857 \\
7 & 40.360 & 0.768 \\
8 & 41.710 & 0.739 \\
9 & 42.239 & 0.720 \\
10 & 42.999 & 0.700 \\
\hline
\end{tabular}

Notes. The substructure test was performed for various group sizes $N_{\text {loc }}$, and $\Delta_{\text {DS }}$ was calculated according to Eqs. (3) and (4) from Sect. 3.4. The listed probability $P$ was calculated from Monte Carlo simulations and rejects substructure in cluster $\mathrm{S} 1101$ with probilities $\geq 70 \%$, depending on the local group size.

Following Dressler \& Shectman (1988), the value of Eq. (3) would exceed the number of all member galaxies $\left(N_{\mathrm{mem}}\right)$ for systems with substructure. We calculated $\Delta_{\mathrm{DS}}$ for our sample of 58 cluster member galaxies by varying group sizes, $5 \leq N_{\text {loc }} \leq$ 10. The results are listed in Table 1 . Despite the fact that according to Pinkney et al. (1996) $N_{\text {loc }} \sim \sqrt{N_{\text {mem }}}$, for all considered values of $N_{\text {loc }}$, the resulting $\Delta_{\mathrm{DS}} / N_{\mathrm{mem}}$ turned out to be well below unity. The test therefore yielded no evidence for the presence of substructure in the cluster S1101.

However, a method more robust that using the "critical value" of unity persists in analysing the probability of $\Delta_{\mathrm{DS}}$, sim exceeding the observed $\Delta_{\mathrm{DS}}$, obs. According to Dressler \& Shectman (1988) $\Delta_{\mathrm{DS}}$, sim is calculated from an artificial sample of randomly reassigned redshifts to the galaxy positions in the sample, the so-called "Monte Carlo shuffling". The randomization process of the galaxy systems destroys any true correlation between position and velocity within those simulated clusters. Accordingly, the probabilities can be computed using

$P=\frac{f\left(\Delta_{\mathrm{DS}, \mathrm{sim}}>\Delta_{\mathrm{DS}, \mathrm{obs}}\right)}{n_{\mathrm{sim}}}$,

where $\Delta_{\mathrm{DS}}$, obs is derived from Eqs. (2) and (3) for the observed sample, while $\Delta_{\mathrm{DS}}$, sim is computed for the sample of the reshuffled members. Only systems with $\Delta_{\mathrm{DS}}$, sim larger than the corresponding observed value contribute to the function $f$ in the probability $P$ given by Eq. (4). For systems with clear substructures $\Delta_{\mathrm{DS}, \text { sim }}>\Delta_{\mathrm{DS}}$, obs is unlikely performed by randomizations and the probability takes low values. Hence we consider a probability of $P<0.01$ (corresponding to $<1 \%$ rejection of substructure) as a clear indication of substructure.

We calculated $P$ using $n_{\mathrm{s}}=100000$ realizations to randomly shuffle our data for different local group sizes (5 to 10); the results are given in Table 1. We find no evidence of substructures in S1101 using either the method based on $\Delta_{\mathrm{DS}}$ or the method based on $P$. S1101 is rather relaxed compared to, for example, the cluster RXC J1504.1-0248 (R1504), for which Zhang et al. (2012) found $P=0.06$ and with evidence of an infall group at about $r_{500}$.

\subsection{Dynamical analysis using the caustic method}

As mentioned in Kaiser (1987), cluster member galaxies tend to form a "trumpet shape" in the project cluster centric distance versus velocity diagram owing the proportionality between the maximum allowed velocity, the escape velocity relative to the cluster, and the enclosed cluster mass. Galaxies with velocities 
Table 2. Properties of the galaxy cluster S1101.

\begin{tabular}{|c|c|}
\hline \multicolumn{2}{|c|}{ Spectroscopic properties (58 galaxies) } \\
\hline$z_{\mathrm{cl}}(58)$ & $0.05601 \pm 0.00027$ \\
\hline$\sigma_{\mathrm{cl}}(58)$ & $\left(574_{-36}^{+38}\right) \mathrm{km} \mathrm{s}^{-1}$ \\
\hline$\sigma_{\text {pas }}(40)$ & $\left(593_{-63}^{+70}\right) \mathrm{km} \mathrm{s}^{-1}$ \\
\hline$r_{200}$ & $\left(1.169_{-0.093}^{+0.097}\right) \mathrm{Mpc}$ \\
\hline$M_{200}$ & $\left(1.92_{-0.42}^{+0.52}\right) \times 10^{14} M_{\odot}$ \\
\hline$r_{500}^{\text {caustic }}$ & $(0.715 \pm 0.005) \mathrm{Mpc}$ \\
\hline$M_{500}^{\text {caustic }}$ & $(1.10 \pm 0.72) \times 10^{14} M_{\odot}$ \\
\hline$r_{200}^{\text {caustic }}$ & $(1.033 \pm 0.005) \mathrm{Mpc}$ \\
\hline$M_{200}^{\text {caustic }}$ & $(1.32 \pm 0.93) \times 10^{14} M_{\odot}$ \\
\hline \multicolumn{2}{|c|}{ X-ray properties } \\
\hline$L_{\mathrm{bol}, 500}$ & $(1.17 \pm 0.10) \times 10^{44} \mathrm{erg} \mathrm{s}^{-1}$ \\
\hline$r_{500}$ & $(0.84 \pm 0.02) \mathrm{Mpc}$ \\
\hline$M_{500}$ & $(1.87 \pm 0.10) \times 10^{14} M_{\odot}$ \\
\hline$M_{\text {gas }, 500}$ & $(1.9 \pm 0.13) \times 10^{13} M_{\odot}$ \\
\hline$r_{200}^{\diamond}$ & $\left(1.37_{-0.18}^{+0.26}\right) \mathrm{Mpc}$ \\
\hline$M_{200}^{\diamond}$ & $\left(2.91_{-0.99}^{+1.99}\right) \times 10^{14} M_{\odot}$ \\
\hline \multicolumn{2}{|c|}{ SZ properties } \\
\hline$M_{500}^{\mathrm{SZ}}$ & $(4.06 \pm 0.92) \times 10^{14} M_{\odot}$ \\
\hline
\end{tabular}

Notes. The number in parenthesis for spectroscopical measures relates to the number of input spectra. We calculated the cluster redshift $\left(z_{\mathrm{cl}}\right)$ and velocity dispersion from all cluster members $\left(\sigma_{\mathrm{cl}}\right)$ and from the fraction of passive galaxies alone $\left(\sigma_{\mathrm{pas}}\right)$, following Beers et al. (1990) as described in Sect. 3.3, and used the values to compute $r_{200}$ and $M_{200}$ following the NFW-fit based scaling in Munari et al. (2013). The density normalized to the critical density, $\rho_{c}\left(z_{\mathrm{cl}}\right)$, was estimated for all radii of Eq. (10), and used to derive the radii and masses from the caustic mass profile for $\Delta=500$ and 200, respectively. In the middle part X-ray properties are listed. $L_{\mathrm{bol}, 500}$ is the cool-core corrected X-ray luminosity. $M_{\text {gas } 500}$ is the gas mass. $M_{500}$ and $r_{500}$ are the total mass and cluster radius derived from the gas mass distribution using the gas mass vs. mass scaling relation. Those X-ray measurements are based on the combined XMM-Newton and ROSAT data from Zhang et al. (2011). Values with diamond superscription are the X-ray results from Reiprich \& Böhringer (2002) using only ROSAT pointed observations.

beyond the "trumpet shape" are not considered to be bound to the cluster potential well and are, therefore, not considered cluster members. The border of the "trumpet shape" defines roughly the maximum velocity scatter within the cluster, and is known as the so-called "caustic", an estimator of the escape velocity for the halo matter distribution with a spherical symmetry. Higher member statistics allow for a more complete sampling of the caustic, which yields more robust measurements of the underlying dark matter distribution of the cluster (e.g. Diaferio \& Geller 1997). The method to measure the caustic is described in detail, for example in Diaferio (1999) and Geller et al. (1999). A good summary and detailed application can also be found in Serra et al. (2011).

The algorithm of the caustic method uses a 2D adaptive kernel to calculate a smoothed density distribution from the phase space coordinates of the input galaxies. In this context, the phase-space spanned along the projected distance and line-ofsight velocity of the galaxies with respect to the cluster centre and its mean velocity, respectively. By definition, the method can be used to identify the membership of galaxies based on the density distribution, which forms a critical curve. In addition, as the curve is an estimator of the escaping velocity, this curve can be used to derive the mass profile of the cluster halo.
The mathematical basics of this method can be found in Silverman (1986) and the 1D approach is outlined in Pisani (1993), whereas the multi-dimensional extension is developed in Pisani (1996).

The input sample for the caustic analysis is based on all galaxies that fulfil $\left|c z_{\mathrm{i}}-c z_{\mathrm{cl}}\right| \leq 4000 \mathrm{~km} \mathrm{~s}^{-1}$, where $z_{\mathrm{cl}}$ is the cluster redshift calculated from the biweight estimator of location (Beers et al. 1990, compare Table 2). In the following, we use our own implementation of the caustic code. Nevertheless, we were fortunate to validate our results against available routines (The Caustic App - Ana Laura Serra \& Antonaldo Diaferio, private communication) and point out major differences below.

We first computed the projected radial distance of each galaxy and its line-of-sight velocity in the cluster rest frame. Contrary to the procedures described in Serra et al. (2011) and Diaferio (1999), we did not weight or average the galaxy positions to derive the cluster centre but for simplicity used the BCG position (23:13:58.60, -42:43:39.0) as the reference cluster centre. Since the BCG of the cluster S1101 is a large and luminous central dominant galaxy and S1101 is rather relaxed, we argue that its position is a good proxy for the global centre of the dark matter halo, which is a fact also discussed in Beers \& Geller (1983).

We used the following formulas from Zhang et al. (2012) to compute projected distance and velocity in the cluster rest frame: $r=D_{\mathrm{a}} \sin \theta$ and $v=\left(c z-c z_{\mathrm{cl}} \cos \theta\right) /\left(1+z_{\mathrm{cl}}\right)$, respectively, for all galaxies. Here, $z_{\mathrm{cl}}, D_{\mathrm{a}}, \theta$, and $z$ are the cluster redshift, angular diameter distance at the cluster redshift, angular separation from the cluster centre and galaxy redshift. Observables and uncertainties were rescaled to match the same units (Mpc) throughout the computation.

Now the 2D density distribution was calculated according to

$f_{q}(\boldsymbol{x})=\frac{1}{N} \sum_{i=1}^{N} \frac{1}{h_{i}^{2}} K\left(\frac{\boldsymbol{x}-\boldsymbol{x}_{i}}{h_{i}}\right)$,

where $\boldsymbol{x}=(r, v)$ is the vector of our input data, whose components are weighted by the local smoothing length $h_{i}$, to get reasonable relative scales between radius and velocity. The value $N$ denotes the number of data points taken into account. The index $q$ is defined as $q=\sigma_{v} / \sigma_{r}$, indicates the relation between the measurement uncertainties in velocity and radius, and will be used later as input for Eq. (7). The input coordinates have to be rescaled in a manner that $q$ is in the acceptable range of 10 to 50 (e.g. Diaferio 1999). The kernel $K$, is defined as:

$K(t)= \begin{cases}4 \pi^{-1}\left(1-t^{2}\right)^{3}, & t<1 \\ 0, & \text { otherwise }\end{cases}$

with $t=\frac{\boldsymbol{x}-\boldsymbol{x}_{i}}{h_{\mathrm{i}}}$. To derive absolute values for the smoothing length, we start with $h_{\mathrm{i}}=h_{c} h_{\mathrm{opt}} \lambda_{i}$. Here $\lambda_{i}=\sqrt{\gamma f_{1}\left(\boldsymbol{x}_{i}\right)^{-1}}$, with $\log \gamma=$ $\sum_{i} N^{-1} \log f_{1}\left(\boldsymbol{x}_{i}\right)$, where $f_{1}$ can be derived from Eq. (5) by fixing $h_{i}$ for all $i$ to the optimal smoothing length

$h_{\mathrm{opt}}=\frac{6.24}{N^{1 / 6}} \sqrt{\frac{\sigma_{r}^{2}+\sigma_{v}^{2}}{2}}$.

The factor in Eq. (7) is different in the literature, here we adopted the value from Serra et al. (2011).

A minimization of

$M_{0}\left(h_{c}\right)=\frac{1}{N^{2}} \sum_{i=1}^{N} \sum_{j=1}^{N}\left(\frac{1}{h_{j}^{2}} K\right)^{2}-\frac{2}{N(N-1)} \sum_{i=1}^{N} \sum_{j \neq i} \frac{1}{h_{j}^{2}} K$ 
returns the smoothing parameter $h_{c}$. The returned value works fine, in general, with well-sampled cluster populations. Suboptimized scaling can, however, result in global under- or overestimation of the contribution from the considered galaxies, leading to a density distribution $f_{q}$ that is either too fine or oversmoothed, which biases the estimation of the escape velocity and thus mass. The minimization gives $h_{c}=0.5682$ according to our data.

Following the procedure of Diaferio (1999), the position of the caustic in the $(r, v)$-diagram is constrained by $f_{q}(r, v)=\kappa$. We then find the proper $\kappa$ by solving

$S(\kappa, R) \equiv\left\langle v_{\mathrm{esc}}^{2}\right\rangle_{\kappa, R}-4\left\langle v^{2}\right\rangle_{R}=0$.

Equation (9) expresses the balancing of the escape velocity and the velocity dispersion from galaxies within the radius $R$, justified by the assumption of virial equilibrium and quasi-isotropic velocity field in the inner part of the cluster. We encompass $R=0.8958 \mathrm{Mpc}$ and $\left\langle v^{2}\right\rangle=476.23\left(\mathrm{~km} \mathrm{~s}^{-1}\right)^{2}$ from binary tree calculations of our input data as measures for the mean radius and squared velocity dispersion of the galaxies associated with the cluster branch of the binary tree. Descriptions of the binary tree are provided in Serra et al. (2011). The mean escape velocity in Eq. (9) remains the only $\kappa$-dependent quantity and can be derived from $\left\langle v_{\mathrm{esc}}^{2}\right\rangle_{\kappa, R}=\int_{0}^{R} \mathcal{A}_{\kappa}^{2}(r) \varphi(r) \mathrm{d} r / \int_{0}^{R} \varphi(r) \mathrm{d} r$. We use $R$ from the binary tree, $\varphi(r)=\int f_{q}(r, v) \mathrm{d} v$ and the caustic amplitude $\mathcal{A}_{\kappa}(r)=\min \left\{\left|v_{\mathrm{u}}\right|,\left|v_{\mathrm{l}}\right|\right\}$. The parameters $v_{\mathrm{u}}$ and $v_{\mathrm{l}}$ are the upper and lower velocity boundary solutions of $f_{q}(r, v)=\kappa$ (which could be determined to $\kappa=0.0016461$ ) for $r$ running from 0 to $R$. The cluster mass within an enclosed radius can be derived from the caustic amplitude as

$G M(\leq r)=\mathcal{F}_{\beta} \int_{0}^{r} \mathcal{A}^{2}(x) \mathrm{d} x$,

with $G$ denoting the gravitational constant and $\mathcal{F}_{\beta}$ the velocity anisotropy. Following Serra et al. (2011), we assume the velocity anisotropy to be independent on the radius, as a firstorder approximation, and hence chose $\mathcal{F}_{\beta}=0.7$. An expression for the error of the mass estimate was computed using $\delta M_{\mathrm{i}}=$ $\sum_{j=1}^{i}\left|2 m_{j} \delta \mathcal{A}\left(r_{j}\right) / \mathcal{A}\left(r_{j}\right)\right|$, where $\delta \mathcal{A}(r) / \mathcal{A}(r)=\kappa / \max \{f(r, v)\}$ is the relative error and $m_{j}$ the mass of a shell at fixed radius.

The caustic mass estimates of the cluster S1101 for $r_{500}^{\text {caustic }}$ and $r_{200}^{\text {caustic }}$ are $M_{500}^{\text {caustic }}=(1.10 \pm 0.72) \times 10^{14} M_{\odot}$ and $M_{200}^{\text {caustic }}=$ $(1.32 \pm 0.93) \times 10^{14} M_{\odot}$, respectively, and are also listed in Table 2. The lower part of Fig. 4 presents the computed mass distribution out to a cluster-centric radius of $\sim 1.6 \mathrm{Mpc}$. In the top panel of Fig. 4 we show the density distribution $f_{q}$ as greyscale and the critical curve $\left(f_{q}=\kappa\right)$, as thick grey contour. The critical curve shows the caustic (blue dashed curve) that defines member galaxies as those in it. Galaxies with their projected distances below or above $R=0.8958 \mathrm{Mpc}$ are shown as red dots or black diamonds, respectively. The literature values included in the study (highlighted as green diamonds) appear as a box shape (a constant velocity dispersion approximately along the radial distance) in the diagram, explaining the underestimation of the velocity dispersion in Zhang et al. (2011) for the cluster S1101. The increased sample of cluster members in this work facilitates the discovery of the "trumpet shaped" caustic expected for a relaxed galaxy cluster. The mean values of the caustic mass, $M_{200}^{\text {caustic }}=(1.32 \pm 0.93) \times 10^{14} M_{\odot}$, and the mass from the velocity dispersion based on the scaling, $M_{200}=\left(1.92_{-0.42}^{+0.52}\right) \times 10^{14} M_{\odot}$, (see also Table 2 or lower panel of Fig. 4) agree well within the calculated errors. Nevertheless, the caustic mass may be underestimated because statistical undersampling of cluster galaxies especially at large cluster centric radii could cause underestimation of the caustic amplitude.

We tested a possible bias in the mass estimate introduced from the fact that our input data consist of only preselected cluster members (see Sect. 3.3), while established implementations of the caustic code do not make assumptions on membership beforehand. In the following, we increased the sample by including additional galaxies from our spectroscopic galaxy sample at $0<z \leq 1.0$. All steps computing the caustic were repeated. The resulting density distribution is slightly different from the previous one due to the presence of fore- and background galaxies. Nevertheless, the caustic method still robustly identifies as the members the same galaxies as those in Fig. 4. This confirms that the caustic method, in which the member statistics only impact the precision of the caustic mass distribution, can distinguish cluster members from interlopers well (see also Serra \& Diaferio 2013). We calculated the contrast density $(\Delta)$ distribution and found that the cluster radii and masses at $\Delta=[500,200]$ vary less than $20 \%$ between the two samples of the input catalogues. This is reasonably caused by the difference in the optimal smoothing and the critical value of the density distribution.

\section{X-ray data analysis and results}

The XMM-Newton and ROSAT X-ray data analysis was detailed in Zhang et al. (2011). In the surface brightness analysis, we directly converted the ROSAT surface brightness profile to the XMM-Newton count rate using the best-fit spectral model obtained from the XMM-Newton data. We then combined the $X M M-N e w t o n$ surface brightness profile within the truncation radius, where the XMM-Newton has a $S / N$ of $\sim 3$, with the ROSAT converted surface brightness profile beyond the truncation radius for further analysis.

The X-ray luminosity is estimated by integrating the X-ray surface brightness. At $3 \sigma$ significance, the surface brightness profile was detected beyond $r_{500}$ combining XMM-Newton and ROSAT data. In practice, we estimated the total count rate from the background-subtracted, flat-fielded, point source-subtracted, and point spread function-corrected surface brightness profile in the $0.7-2 \mathrm{keV}$ band. We converted this to X-ray luminosity using the best-fit "mekal" model in XSPEC of the spectra extracted in the aperture within the XMM-Newton field of view defined in Sect. 3.2 in Zhang et al. (2009). Combined observations of ROSAT and XMM-Newton in Zhang et al. (2011) yield consistent total X-ray luminosity measurements as those based on ROSAT observations alone in Reiprich \& Böhringer (2002) within the $15 \%$ level as shown in Fig. A.1 in Zhang et al. (2011). This $15 \%$ difference is a result of introducing the corrections for point sources and substructures in Zhang et al. (2011).

The value in this work is a cool-core corrected measurement $\left(L_{\mathrm{bol}, 500}=(1.17 \pm 0.10) \times 10^{44} \mathrm{erg} \mathrm{s}^{-1}\right)$, as defined in Sect. 2.2.2 in Zhang et al. (2011), which is thus much lower than the total $\mathrm{X}$-ray luminosity. With the cool-core corrected measurements we can suppress the scatter from the cool core, which allows us to focus on the scatter due to other facts in studying the $L_{\mathrm{bol}}-\sigma$ scaling relation.

As detailed in Sect. 2.2.1 in Zhang et al. (2011), the cluster radius $r_{500}$ was determined from the $\mathrm{X}$-ray gas mass profile through the gas mass versus total mass scaling relation under the assumption of spherical symmetry. Pratt et al. (2009) showed a tight scaling between gas mass and total mass for a sample of 


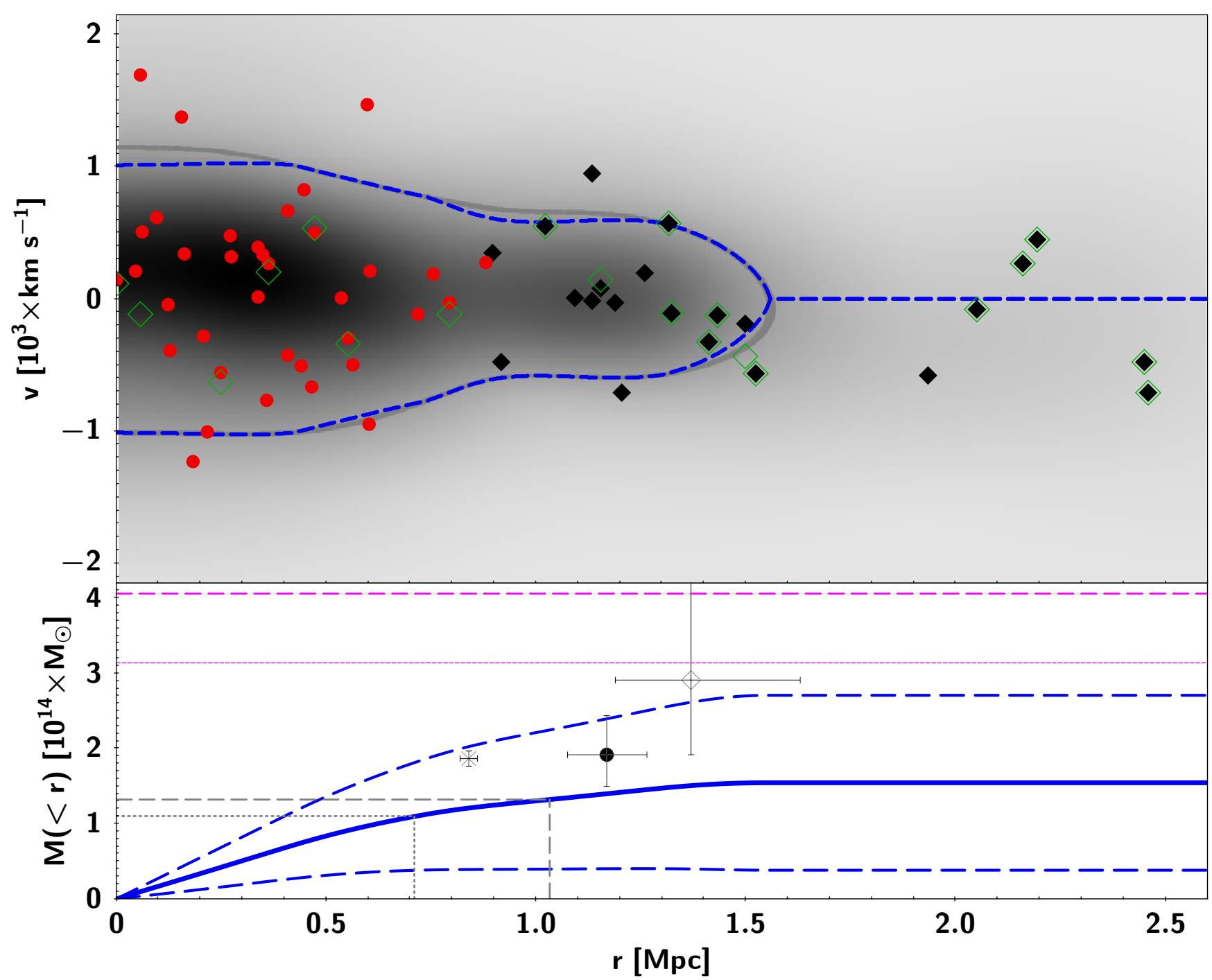

Fig. 4. Upper panel: diagram of relative line-of-sight velocity vs. projected cluster centric distance of the candidate member galaxies in S1101. Grey shadow shows the $f_{q}(r, v)$ contours. The thick grey curve highlights the equal-density curve of $f_{q}(r, v)=\kappa$. Red circles and black diamonds indicate galaxies within and beyond $r=0.896 \mathrm{Mpc}$ (used for constraining $\kappa$ ), respectively. The blue dashed curve refers to the estimated caustic of the cluster, hence all galaxies with their velocities beyond are excluded from the member sample in this method. Green open diamonds highlight cluster members previously known in the literature. Lower panel: mass profile of S1101. The mass profile from the caustic method (see Sect. 3.5) is shown as a blue thick curve, where the blue dashed curves show the corresponding $1 \sigma$ error estimate. The grey dashed and dotted lines trace the measured positions of $r_{\Delta}^{\text {caustic }}$ and $M_{\Delta}^{\text {caustic }}$ for $\Delta=\{500,200\}$, respectively, according to the caustic mass distribution. For comparison, we overplot the dynamical mass $M_{200}$ derived from the velocity dispersion according to Munari et al. (2013) as large black dot, the X-ray mass estimates $M_{500}$ (Zhang et al. 2011) and $M_{200}^{\diamond}$ (Reiprich \& Böhringer 2002) as black X and open diamond, respectively, and the SZ mass proxy as a dashed line with its lower error interval as dotted lines in magenta. Values of all mass proxies are also given in Table 2.

41 groups and clusters as follows:

$E(z)^{\frac{3}{2}} \ln \left(\frac{M_{\text {gas }, 500}}{M_{500}}\right)=-2.37+0.21 \ln \left(\frac{M_{500}}{2 \times 10^{14} M_{\odot}}\right)$.

We infer the total mass profile of S1101, $M(<r)$, from the measured gas mass profile, $M_{\mathrm{gas}}(<r)$, to find the radius, $r_{500}$, which fulfils

$M_{500}=\frac{4 \pi}{3} 500 \rho_{c}(z) r_{500}^{3}$.

The resulting cluster radius is $r_{500}=(0.84 \pm 0.02) \mathrm{Mpc}$, within which the gas mass is $M_{\text {gas, } 500}=(1.90 \pm 0.13) \times 10^{13} M_{\odot}$ and the total cluster mass is $M_{500}=(1.87 \pm 0.10) \times 10^{14} M_{\odot}$. The errors of $r_{500}$ and $M_{500}$ are only based on the error of $M_{\mathrm{gas}, 500}$ which do not include the intrinsic scatter of the scaling relation.
For comparison in Fig. 4, we also include $M_{200}^{\diamond}$ (see also Table 2) with an open diamond symbol, as X-ray mass estimate under the assumptions of spherical symmetry and hydrostatic equilibrium, derived by Reiprich \& Böhringer (2002) from ROSAT pointed observations. It is not clear that the Reiprich \& Böhringer (2002) mass overestimates the true mass since only a single beta model was fitted to the surface brightness profile. If the profile actually steepens at large radii then this effect may roughly compensate the isothermal assumption. The X-ray measurements discussed above are also listed in Table 2. Additionally we plot these radii and masses in the lower panel of Fig. 4 for comparison. When considering at least $10 \%$ scatter in the gas mass versus total mass relation (e.g. Okabe et al. 2010), the mass, $M_{500}$, from Zhang et al. (2011) agrees rather well with the virial mass. The mass, $M_{200}^{\diamond}$, from Reiprich \& Böhringer (2002) based on an isothermal gas model 
slightly overestimates the mass, but still agrees with the virial estimate $M_{200}$ within the uncertainties (Table 2).

\section{South Pole Telescope detection of S1101}

Modern surveys with millimeter or submillimeter telescopes are able to measure tiny deviations in the cosmic microwave background (CMB). When photons from the CMB undergo inverseCompton scattering while passing by the intracluster medium (ICM) of galaxy clusters, an effect known as the SZ effect (Sunyaev \& Zel'dovich 1972), a relative temperature difference with respect to the mean CMB temperature is expected. This effect has a strong correlation with the mass of the ICM and puts no redshift dependent luminosity constraints on the observability, as for example in X-rays. Theoretically, SZ effect based observations are able to detect a mass-limited sample of galaxy clusters.

The South Pole Telescope (SPT; Carlstrom et al. 2011) is a $10 \mathrm{~m}$ telescope in Antarctica that observes the southern sky in three bands centred at 95, 150, and $220 \mathrm{GHz}$. We found S1101 in their public catalogue of the $2500 \mathrm{deg}^{2}$ data (detailed in Bleem et al. 2015). It is listed as SPT-CL J2313-4243 with a mass derived from the SZ detection significance using the scaling relation, $M_{500}^{\mathrm{SZ}}=(4.06 \pm 0.92) \times 10^{14} M_{\odot}$, which is much higher than the caustic as well as X-ray masses.The scaling relation between the SZ detection significance and total mass is not particularly tight for deriving a robust mass estimate. Indeed, the provided $M_{500}^{\mathrm{SZ}}$ for S1101 is much larger than the caustic and the X-ray masses, still Bleem et al. (2015) consider that masses derived for low redshift clusters are possibly biased low. We list the mass measurement in Table 2 and plotted it as a dashed magenta line in comparison with the caustic mass profile of S1101 in Fig. 4.

\section{Discussion on background structure}

As mentioned in Sect. 3.1, our spectroscopic dataset displays an overdensity of background galaxies at approximately twice the cluster redshift (see Fig. 2). We thus examined the spectroscopic redshift catalogue comprising 191 galaxies at $0<z<0.2$ (from now on SQ), which encloses cluster members and possible background structures. In order to detect substructures within this broad redshift range, we applied the DS test (as described in Sect. 3.4) with $N_{\text {loc }}=10$, to this catalogue.

In Fig. 5, we show the sky positions of these galaxies, where their symbol sizes scale with $\exp \left(\delta_{i}^{2}\right)$. There are two clumps that have high probabilities of being substructures. As highlighted by the colour-coded redshift information, the purple circles represent the dense core of galaxy cluster S1101, while green circles indicate a potential background structure at $0.09<z<0.11$. We made an attempt to assign membership of the galaxies to the tentative background structure using the following procedure:

1) We selected the galaxies with the highest $\delta_{\mathrm{i}}$ at larger redshift than the cluster S1101 (compare colour-coding in Fig. 5) and calculated the mean redshift, $\bar{z}$, of those 7 galaxies.

2) With a gap clipping of $\left|c \bar{z}-c z_{\mathrm{S} 0}\right| \leq 4000 \mathrm{~km} \mathrm{~s}^{-1}$ around $\bar{z}$, a first outlier-rejected sample called S1 was derived.

3) Application of the biweight estimators combined with the $\left|c z-c \overline{z_{\mathrm{S} 1}}\right| \leq 3 \sigma_{\mathrm{S} 1}$ clipping in iterative manner resulted in the final sample of 27 galaxies for this background structure, called the S2 sample.

4) Final redshift and velocity dispersion of the S2 sample were computed using the biweight estimators of location and scale.

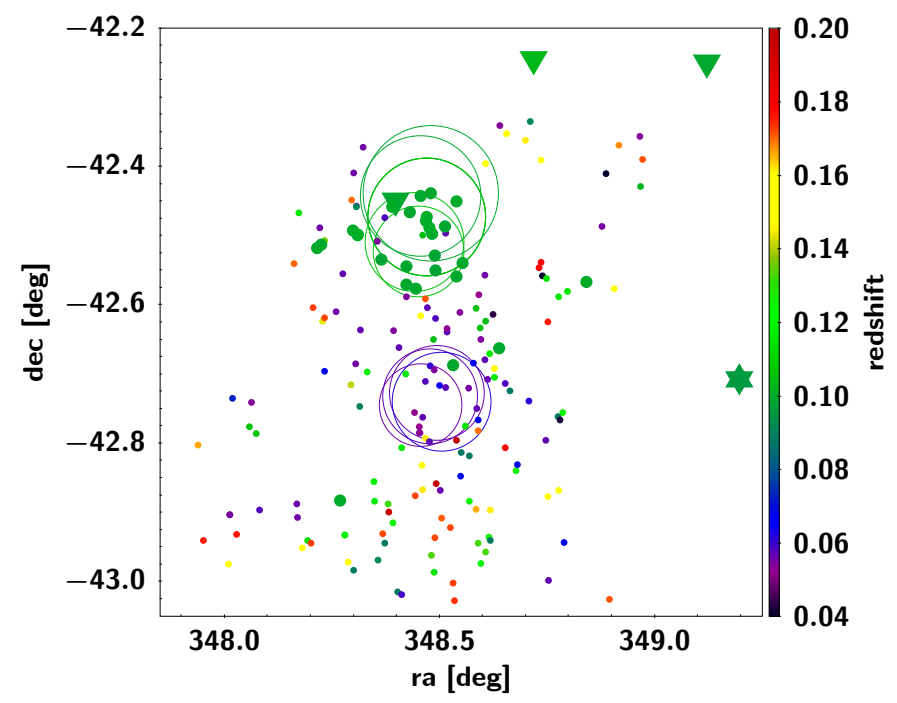

Fig. 5. Dressler-Shectman plot of our spectroscopic sample SQ. Symbol size scales with $\exp \left(\delta_{i}^{2}\right)$ above a threshold of $\delta_{i}=3$, below that value galaxies are shown as simple dots, and the sample S2 is emphasized by larger green dots. Filled triangles and the filled star symbolize the central regions of galaxy groups and one galaxy cluster found in the vicinity of our survey field, as listed in the NED. The colour of each symbol corresponds to the redshift of the respective source.

These steps follow the same method we applied when selecting member galaxies for the cluster S1101 (compare Sect. 3.3).

We note that by using the fixed gapper method with the typical gap of $1000 \mathrm{~km} \mathrm{~s}^{-1}$, the whole sample S1 is regarded as a group or cluster. Referring to Fig. 6, in addition to the objects within the blue dotted lines (the final clipping for sample S2) the overdensity of 7 galaxies centred at $z \sim 0.105$ would contribute to the member population as well. The fixed gap may, however, may be too large for such low mass systems. The additional overdensity may indicate some structure that may be connected with, but not directly belong, to S2. It is still possible that both systems are interacting gravitationally, but that is not part of this discussion.

We derive $z_{\mathrm{S} 2}=0.09974 \pm 0.00017$ and $\sigma_{\mathrm{S} 2}=$ $\left(195_{-39}^{+49}\right) \mathrm{km} \mathrm{s}^{-1}$ for the S2 sample. Following Munari et al. (2013), we obtained a mass of $M_{200}=\left(0.93_{-0.49}^{+1.00}\right) \times 10^{13} M_{\odot}$, which indicates that S2 is a galaxy group, despite its high number of member galaxies.

A query in the NED for group or cluster counterparts resulted in one cluster and several groups of galaxies near the cluster S1101. The cluster is visualized as a yellow star, while the groups are shown as filled triangles in Fig. 5. The galaxy group that coincides best with our findings is LCLG -42 230 from the "Las Campanas Loose Group" catalogue and has a published spectroscopic redshift of 0.0991 (Tucker et al. 2000). In the Las Campanas Redshift Survey, the redshift was only calculated from 6 galaxies, compared to 27 galaxies for this work. We measured a sky distance of $\sim 3.69^{\prime}$ between the S2 mean coordinates and the position in the LCLG catalogue, corresponding to a distance of $\sim 406 \mathrm{kpc}$ at the redshift of the group. This distance is comparable to the virial radius of S2. The 27 galaxies serendipitously found in our data may well belong to LCLG -42 230.

The scaling relation in Fig. 7 yields an X-ray luminosity of $\sim 4 \times 10^{41} \mathrm{erg} \mathrm{s}^{-1}$ for this group from its measured $\sigma$. This corresponds to a flux that is more than three magnitudes lower than 


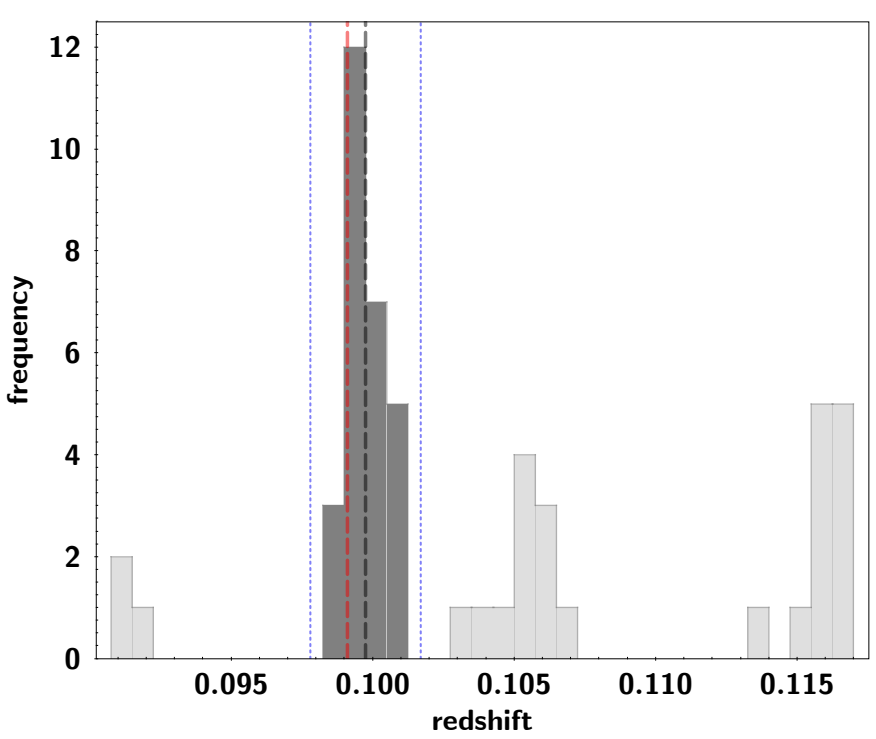

Fig. 6. Redshift histogram of the sample $\mathrm{S} 1$ around $z \sim 0.1$. The dotted blue vertical lines refer to the borders of the final clipping, while the actual members of the sample S2 are shown as a dark shaded region. The redshift of galaxy group LCLG -42230 is indicated by a black and red vertical line, based on our sample S2 and previous work of Tucker et al. (2000), respectively. The definition of S1 and S2 are given in Sect. 6; the corresponding data are listed in Appendix A.

that of S1101. It can therefore not cause any significant bias in the X-ray measurement of S1101.

\section{Conclusions}

We performed multi-object spectroscopic follow-up observations of the galaxies in the S1101 field in order to increase the member statistics for the dynamical study of this field. The combined sample of our survey and the redshifts from the NED yield 58 or 42 cluster member galaxies, using the member identification method of Beers et al. (1990) or the caustic method, respectively.

The number of 47 confirmed (from our VIMOS spectra only) of 392 candidate cluster members, which corresponds to $(\sim 12 \%)$, might appear relatively low. Considering galaxies with magnitudes $R<18$ mag the rate between confirmed and observed sources still is 24 versus 69 , which corresponds to $\sim 35 \%$. Observational restrictions demanded to also include fainter targets but increased the influence of photometric uncertainties. Despite unfavourable conditions during pre-imaging a sound sample of member galaxies could be established by taking advantage of the survey capabilities offered by VIMOS. Large MOS capabilities will however not necessarily be available for future large-scale automized surveys, underlying the importance of a suitable preselection.

The 2D dynamical substructure tests indicate no ongoing merging activities in S1101; this finding is also supported by the large percentage of passive galaxies residing in the cluster centre and the absence of severe inhomogeneities in the available X-ray data.

The increased number of member galaxies with spectroscopic redshifts and the relaxed cluster structure indicated in our $2 \mathrm{D}$ test ensure robust constraints on the velocity dispersion and virial mass estimates. The recovery of the "trumpet shape" in the $v-r$ plot (Fig. 4) indicates an improved completeness of the confirmed cluster population and hence a reduced bias in $\sigma$

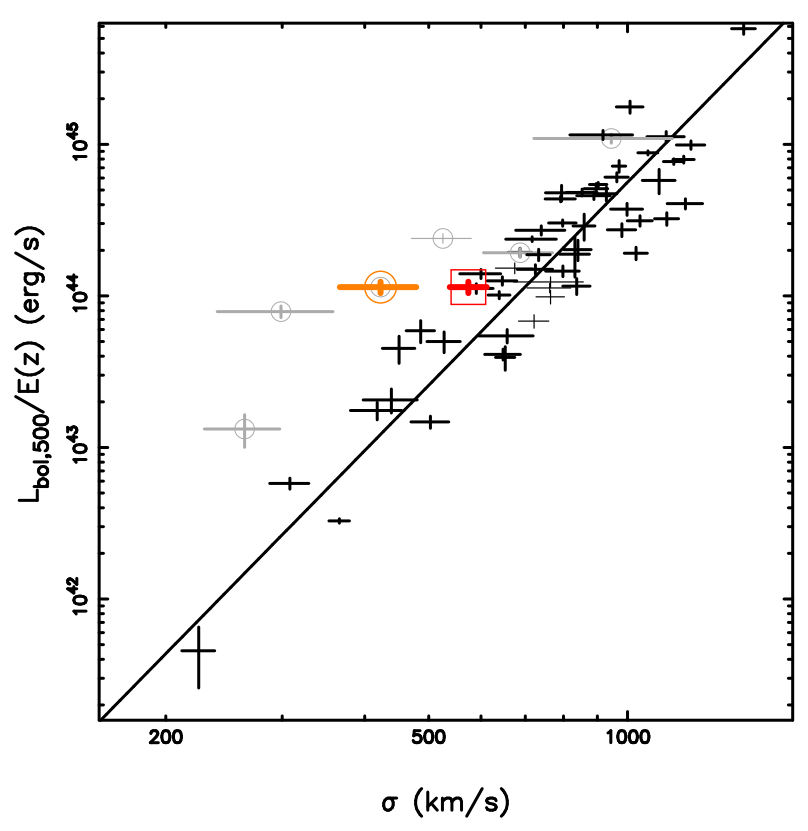

Fig. 7. $L_{\mathrm{bol}}^{\mathrm{cocc}}-\sigma$ diagram of the HIFLUGCS clusters, in which the best fit is for those 56 clusters with more than 45 spectroscopic members per cluster. The orange circle and red box show the original (Zhang et al. 2011) and the revised values from this work, respectively, for S1101. Clusters with less than 45 spectroscopic members are shown as grey circles.

with respect to the previous study of Zhang et al. (2011). Using the cluster velocity dispersion, $\sigma$, we updated the position of S1101 in the $L_{\text {bol }}-\sigma$ diagram (Fig. 7) with respect to the work of Zhang et al. (2011). We confirmed the hint in Zhang et al. (2011) based on the simulations sample that the severe deviation of S1101 from the indicated scaling relation therein is mostly due to the velocity dispersion measurement with low member statistics. This result adds confidence for the interpretation as a bias due to a small number of member galaxies. Given the ongoing efforts for large-scale cluster mass calibration with $\sim 20$ members in a cluster, for example, SPIDERS (SPectroscopic IDentification of ERosita Sources) and 4MOST (4-m Multi-Object Spectroscopic Telescope) for the eROSITA clusters (e.g. Merloni et al. 2012), it is useful to determine a correction factor based on our whole sample in Zhang et al. (2011). A spectroscopic follow-up of the remaining outlier clusters in our sample in the near future would help to realize this goal.

The mass estimate from the velocity dispersion, $M_{200}=$ $\left(1.92_{-0.42}^{+0.52}\right) \times 10^{14} M_{\odot}$, agrees with the available X-ray mass proxies (Zhang et al. 2011; Reiprich \& Böhringer 2002) within the uncertainties. The SZ mass proxy (Bleem et al. 2015) is much higher than the remaining mass estimates, namely the dynamical, X-ray , and caustic-based masses (compare Table 2 and Fig. 4). This might be due to the large scatter in the scaling relation between the SZ detection significance and total mass they used. The caustic method makes no assumption on the dynamical state of the cluster, and traces the enclosed cluster mass as a function of radius, solely by positions and redshifts of member galaxies. The caustic mass profile might slightly underestimate the cluster mass given a rather small sample of cluster galaxies distributed up to a large projected distance of $2.5 \mathrm{Mpc}$. We therefore note that the caustic method is in principle able to trace the cluster mass out to large radii $\left(>2 r_{200}\right)$, but relies on high member statistics in order to reach high precision (200 or more; see Serra et al. 2011). The member statistics of S1101 in this study is 
still too low to deliver a high-precision application of the caustic method.

In addition to the population of cluster galaxies, the redshift distribution revealed an apparent overdensity at $z \sim 0.1$. The 2D dynamical substructure tests using galaxies at $0<z<0.2$ and the further analysis of those galaxies, suggested a group of 27 galaxies with $z_{\mathrm{S} 2}=0.09974 \pm 0.00017$ (see Sect. 6). We measured a velocity dispersion of $\sigma_{\mathrm{S} 2}=\left(195_{-39}^{+49}\right) \mathrm{km} \mathrm{s}^{-1}$ for this group, which yields $M_{200}=\left(0.93_{-0.49}^{+1.00}\right) \times 10^{13} M_{\odot}$ following Munari et al. (2013). The resulting X-ray luminosity according to our scaling relation is less than $1 \%$ of that of $\mathrm{S} 1101$. No significant bias from this group should be expected in the X-ray luminosity estimate of S1101.

Acknowledgements. We thank the anonymous referee for constructive criticism that helped to improve the readability of the paper. We thank A. Biviano, H. Böhringer, and J. Dietrich for their numerous suggestions that helped this work. Furthermore, we thank A. L. Serra and A. Diaferio for the communication on the caustic method and kindness to cross-check our results with their code, as well as Heinz Andernach for his hint on a case with a lack of clearity. The Guide Star Catalogue-II is a joint project of the Space Telescope Science Institute and the Osservatorio Astronomico di Torino. Space Telescope Science Institute is operated by the Association of Universities for Research in Astronomy, for the National Aeronautics and Space Administration under contract NAS526555. The participation of the Osservatorio Astronomico di Torino is supported by the Italian Council for Research in Astronomy. Additional support is provided by European Southern Observatory, Space Telescope European Coordinating Facility, the International GEMINI project and the European Space Agency Astrophysics Division. We have made use of VLT/VIMOS observations taken with the ESO Telescope at the Paranal Observatory under programme 087.A0096 and WFI observations partially supported by the Deutsche Forschungsgemeinschaft (DFG) through Transregional Collaborative Research Centre TRR 33. The XMM-Newton project is an ESA Science Mission with instruments and contributions directly funded by ESA Member States and the USA (NASA). The $X M M-N e w t o n$ project is supported by the Bundesministerium für Wirtschaft und Technologie/Deutsches Zentrum für Luft- und Raumfahrt (BMWI/DLR, FKZ 50 OX 0001) and the Max-Planck Society. This research has made use of the NASA/IPAC Extragalactic Database (NED) which is operated by the Jet Propulsion Laboratory, California Institute of Technology, under contract with the $\mathrm{Na}$ tional Aeronautics and Space Administration. We used the version 13SEPpl1.4 of ESO-MIDAS for data reduction and STILTS ${ }^{1}$ for table processing and plotting of figures. Y.Y.Z. acknowledges support by the German BMWi through the Verbundforschung under grant 50 OR 1506. T.H.R. acknowledges support from the DFG through the Heisenberg research grant RE 1462/5.

\section{References}

Baade, D., Meisenheimer, K., Iwert, O., et al. 1999, The Messenger, 95, 15 Banse, K., Crane, P., Grosbol, P., et al. 1983, The Messenger, 31, 26 Beers, T. C., \& Geller, M. J. 1983, ApJ, 274, 491

Beers, T. C., Flynn, K., \& Gebhardt, K. 1990, AJ, 100, 32

Bertin, E., \& Arnouts, S. 1996, A\&AS, 117, 393

Biviano, A., \& Girardi, M. 2003, ApJ, 585, 205

Biviano, A., Murante, G., Borgani, S., et al. 2006, A\&A, 456, 23

Bleem, L. E., Stalder, B., de Haan, T., et al. 2015, ApJS, 216, 27

Böhringer, H., Schuecker, P., Guzzo, L., et al. 2001, A\&A, 369, 826
Böhringer, H., Schuecker, P., Guzzo, L., et al. 2004, A\&A, 425, 367 Borgani, S., \& Guzzo, L. 2001, Nature, 409, 39

Carlberg, R. G., Yee, H. K. C., Ellingson, E., et al. 1997, ApJ, 485, L13 Carlstrom, J. E., Ade, P. A. R., Aird, K. A., et al. 2011, PASP, 123, 568

de Vaucouleurs, G., de Vaucouleurs, A., Corwin, Jr., H. G., et al. 1991, Third Reference Catalogue of Bright Galaxies, Vol. I: Explanations and references, Vol. II: Data for galaxies between $0^{\mathrm{h}}$ and $12^{\mathrm{h}}$, Vol. III: Data for galaxies between $12^{\mathrm{h}}$ and $24^{\mathrm{h}}$

Diaferio, A. 1999, MNRAS, 309, 610

Diaferio, A., \& Geller, M. J. 1997, ApJ, 481, 633

Dressler, A., \& Shectman, S. A. 1988, AJ, 95, 985

Erben, T., Schirmer, M., Dietrich, J. P., et al. 2005, Astron. Nachr., 326, 432

Evrard, A. E., Bialek, J., Busha, M., et al. 2008, ApJ, 672, 122

Garilli, B., Fumana, M., Franzetti, P., et al. 2010, PASP, 122, 827

Geller, M. J., Diaferio, A., \& Kurtz, M. J. 1999, ApJ, 517, L23

Gladders, M. D., \& Yee, H. K. C. 2005, ApJS, 157, 1

Horne, K. 1986, PASP, 98, 609

Jones, D. H., Read, M. A., Saunders, W., et al. 2009, MNRAS, 399, 683

Kaiser, N. 1987, MNRAS, 227,

Katgert, P., Mazure, A., Perea, J., et al. 1996, A\&A, 310, 8

Le Fèvre, O., Saisse, M., Mancini, D., et al. 2003, in Instrument Design and Performance for Optical/Infrared Ground-based Telescopes, eds. M. Iye, \& A. F. M. Moorwood, Proc. SPIE, 4841, 1670

Mantz, A. B., Allen, S. W., Morris, R. G., et al. 2014, MNRAS, 440, 2077

Maurogordato, S., Cappi, A., Ferrari, C., et al. 2008, A\&A, 481, 593

Merloni, A., Predehl, P., Becker, W., et al. 2012, ArXiv e-prints [arXiv: 1209.3114]

Munari, E., Biviano, A., Borgani, S., Murante, G., \& Fabjan, D. 2013, MNRAS, 430, 2638

Navarro, J. F., Frenk, C. S., \& White, S. D. M. 1997, ApJ, 490, 493

Okabe, N., Zhang, Y.-Y., Finoguenov, A., et al. 2010, ApJ, 721, 875

Pinkney, J., Roettiger, K., Burns, J. O., \& Bird, C. M. 1996, ApJS, 104, 1

Pisani, A. 1993, MNRAS, 265, 706

Pisani, A. 1996, MNRAS, 278, 697

Pratt, G. W., Croston, J. H., Arnaud, M., \& Böhringer, H. 2009, A\&A, 498, 361

Puchwein, E., Sijacki, D., \& Springel, V. 2008, ApJ, 687, L53

Reiprich, T. H., \& Böhringer, H. 2002, ApJ, 567, 716

Saro, A., Mohr, J. J., Bazin, G., \& Dolag, K. 2013, ApJ, 772, 47

Schirmer, M. 2013, ApJS, 209, 21

Schneider, P. 1996, MNRAS, 283, 837

Serra, A. L., \& Diaferio, A. 2013, ApJ, 768, 116

Serra, A. L., Diaferio, A., Murante, G., \& Borgani, S. 2011, MNRAS, 412, 800

Shectman, S. A., Landy, S. D., Oemler, A., et al. 1996, ApJ, 470, 172

Silverman, B. W. 1986, Density estimation for statistics and data analysis (London: Chapman and Hall)

Skrutskie, M. F., Cutri, R. M., Stiening, R., et al. 2006, AJ, 131, 1163

Sunyaev, R. A., \& Zeldovich, Y. B. 1972, Comments on Astrophysics and Space Physics, 4, 173

Taylor, M. B. 2006, in Astronomical Data Analysis Software and Systems XV, eds. C. Gabriel, C. Arviset, D. Ponz, \& S. Enrique, ASP Conf. Ser. 351, 666

Taylor, M. B. 2009, in Astronomical Data Analysis Software and Systems XVIII, eds. D. A. Bohlender, D. Durand, \& P. Dowler, ASP Conf. Ser., 411, 510

Trümper, J. 1992, QJRAS, 33, 165

Tucker, D. L., Oemler, Jr., A., Hashimoto, Y., et al. 2000, ApJS, 130, 237

Vale, C., \& White, M. 2006, New Astron., 11, 207

Weinberg, N. N., \& Kamionkowski, M. 2002, MNRAS, 337, 1269

Zabludoff, A. I., \& Mulchaey, J. S. 1998, ApJ, 496, 39

Zhang, Y.-Y., Reiprich, T. H., Finoguenov, A., Hudson, D. S., \& Sarazin, C. L. 2009, ApJ, 699, 1178

Zhang, Y.-Y., Andernach, H., Caretta, C. A., et al. 2011, A\&A, 526, A105

Zhang, Y.-Y., Verdugo, M., Klein, M., \& Schneider, P. 2012, A\&A, 542, A106

1 http://wWW.starlink.ac.uk/stilts/ 


\section{Appendix A: Spectroscopic targets of LCLG -42 230}

This section lists sky coordinates and redshifts for the sample $\mathrm{S} 1$, contributing to the background structure at redshift $z \sim 0.1$ in the field of view of S1101. The subsample, with considered affiliation to LCLG -42230 , is highlighted as S2 in Col. 4. The sample properties are explained in detail in Sect. 6, coordinates correspond to GSC2.3 sources and the listed redshifts are based on fits to passive and active galaxy templates by EZ.

Table A.1. Sample S1 of the background group at $z \sim 0.1$.

\begin{tabular}{cccc}
\hline \hline RA & Dec & $z$ & Sample \\
\hline $23: 12: 13.8$ & $-42: 46: 36$ & 0.1067 & S1 \\
$23: 12: 17.8$ & $-42: 47: 10$ & 0.1065 & S1 \\
$23: 12: 41.7$ & $-42: 28: 04$ & 0.1166 & S1 \\
$23: 12: 46.5$ & $-42: 56: 27$ & 0.1134 & S1 \\
$23: 12: 51.7$ & $-42: 31: 05$ & 0.1000 & S2 \\
$23: 12: 54.0$ & $-42: 30: 50$ & 0.0997 & S2 \\
$23: 13: 04.7$ & $-42: 52: 59$ & 0.1002 & S2 \\
$23: 13: 12.0$ & $-42: 29: 34$ & 0.1002 & S2 \\
$23: 13: 13.6$ & $-42: 27: 31$ & 0.0914 & S1 \\
$23: 13: 14.3$ & $-42: 29: 59$ & 0.1012 & S2 \\
$23: 13: 14.5$ & $-42: 29: 57$ & 0.1003 & S2 \\
$23: 13: 24.0$ & $-42: 53: 05$ & 0.1167 & S1 \\
$23: 13: 25.9$ & $-42: 58: 08$ & 0.0922 & S1 \\
$23: 13: 27.5$ & $-42: 32: 05$ & 0.0983 & S2 \\
$23: 13: 33.6$ & $-42: 27: 33$ & 0.1001 & S2 \\
$23: 13: 39.1$ & $-42: 48: 23$ & 0.1164 & S1 \\
$23: 13: 41.2$ & $-42: 42: 03$ & 0.1160 & S1 \\
$23: 13: 41.5$ & $-42: 32: 42$ & 0.0992 & S2 \\
$23: 13: 41.8$ & $-42: 34: 15$ & 0.0993 & S2 \\
$23: 13: 43.4$ & $-42: 28: 00$ & 0.0994 & S2 \\
$23: 13: 46.2$ & $-42: 30: 35$ & 0.1056 & S1 \\
$23: 13: 46.9$ & $-42: 34: 38$ & 0.0995 & S2 \\
$23: 13: 48.4$ & $-42: 31: 24$ & 0.1065 & S1 \\
$23: 13: 49.5$ & $-42: 26: 34$ & 0.0993 & S2 \\
$23: 13: 50.8$ & $-42: 30: 00$ & 0.1055 & S1 \\
$23: 13: 52.7$ & $-42: 28: 47$ & 0.1003 & S2 \\
$23: 13: 52.8$ & $-42: 28: 23$ & 0.1006 & S2 \\
$23: 13: 52.8$ & $-42: 28: 25$ & 0.1062 & S1 \\
$23: 13: 54.4$ & $-42: 29: 21$ & 0.1009 & S2 \\
$23: 13: 55.4$ & $-42: 26: 19$ & 0.0996 & S2 \\
$23: 13: 55.8$ & $-42: 29: 51$ & 0.0993 & S2 \\
$23: 13: 56.8$ & $-42: 39: 02$ & 0.1044 & S1 \\
$23: 13: 57.2$ & $-42: 59: 11$ & 0.1149 & S1 \\
$23: 13: 57.6$ & $-42: 31: 44$ & 0.0986 & S2 \\
$23: 13: 57.9$ & $-42: 33: 02$ & 0.0985 & S2 \\
$23: 14: 03.3$ & $-42: 29: 13$ & 0.0992 & S2 \\
$23: 14: 07.7$ & $-42: 41: 17$ & 0.0998 & S2 \\
$23: 14: 09.6$ & $-42: 27: 04$ & 0.1005 & S2 \\
$23: 14: 09.7$ & $-42: 33: 33$ & 0.0996 & S2 \\
$23: 14: 13.1$ & $-42: 32: 23$ & 0.0995 & S2 \\
$23: 14: 14.3$ & $-42: 46: 33$ & 0.1156 & S1 \\
\hline & & &
\end{tabular}

Notes. The subsample S2 is included in S1 but is highlighted for simplicity only as a subgroup (see Col. 4). While S1 corresponds to a simple velocity-clipped sample, S2 is based on biweight estimates and iterative sigma-clipping (see Sect. 6 for details). Galaxies of the latter sample are considered group members of LCLG -42230 . Position in RA and Dec (J2000) is based on GSC2.3 astrometry. The spectroscopic redshift is given in Col. 3 .
Table A.1. continued.

\begin{tabular}{cccc}
\hline \hline RA & Dec & $z$ & Sample \\
\hline $23: 14: 20.5$ & $-42: 36: 21$ & 0.1057 & S1 \\
$23: 14: 22.7$ & $-42: 38: 03$ & 0.1051 & S1 \\
$23: 14: 25.7$ & $-42: 37: 28$ & 0.1039 & S1 \\
$23: 14: 28.1$ & $-42: 40: 16$ & 0.1163 & S1 \\
$23: 14: 30.9$ & $-42: 42: 17$ & 0.1166 & S1 \\
$23: 14: 33.3$ & $-42: 39: 47$ & 0.0994 & S2 \\
$23: 14: 50.7$ & $-42: 20: 06$ & 0.0914 & S1 \\
$23: 14: 59.8$ & $-42: 33: 44$ & 0.1156 & S1 \\
$23: 15: 06.7$ & $-42: 35: 18$ & 0.1157 & S1 \\
$23: 15: 11.5$ & $-42: 34: 52$ & 0.1158 & S1 \\
$23: 15: 22.2$ & $-42: 34: 03$ & 0.1005 & S1 \\
$23: 15: 52.2$ & $-42: 25: 47$ & 0.1029 & S2 \\
\hline
\end{tabular}

\section{Appendix B: Spectroscopic targets of S1101}

Using the iterative sigma-clipping method, as described in Sect. 3.3, our sample of cluster member galaxies for the cluster S1101 comprises 58 galaxies. We list this sample, including sky coordinates, redshift, and a simple spectral classification in Table B.1. The coordinates given in the table correspond to GSC2.3 sources. As a reference we list spectral redshifts from various publications (results from the NED query). Whenever data is available in our sample, we prefer to use our own for the final redshift measurements and spectral classification (see Sect. 3.1). The visibility of the spectral emission lines is decisive for either passive or active classification, corresponding to $\theta$ and 1 in Col. 4 of Table B.1. Those objects, which have no spectra available or have spectral coverage and data quality hampered the decision process, were assigned 99. 
A. Rabitz et al.: Probing the dynamical and X-ray mass proxies of Abell S1101

Table B.1. Sample of spectroscopically confirmed cluster members of S1101.

\begin{tabular}{|c|c|c|c|c|c|}
\hline RA & Dec & $z$ & Type & $R$ & Reference \\
\hline $23: 11: 40.7$ & $-43: 06: 31$ & 0.05694 & 0 & - & Jones et al. (2009) \\
\hline $23: 11: 47.7$ & $-43: 05: 21$ & 0.05571 & 1 & - & Jones et al. (2009) \\
\hline $23: 12: 03.2$ & $-42: 54: 13$ & 0.05532 & 1 & 15.860 & - \\
\hline $23: 12: 15.2$ & $-42: 44: 28$ & 0.05349 & 0 & 17.611 & - \\
\hline $23: 12: 19.9$ & $-42: 53: 50$ & 0.05800 & 0 & 15.518 & Jones et al. (2009) \\
\hline $23: 12: 40.5$ & $-42: 53: 16$ & 0.05602 & 0 & 16.208 & - \\
\hline $23: 12: 41.0$ & $-42: 54: 28$ & 0.05595 & 1 & 18.487 & - \\
\hline 23:12:53.1 & $-42: 29: 19$ & 0.05589 & 1 & 18.837 & - \\
\hline $23: 13: 02.2$ & $-42: 36: 36$ & 0.05589 & 0 & 15.272 & - \\
\hline $23: 13: 06.0$ & $-42: 33: 21$ & 0.05721 & 0 & 18.481 & - \\
\hline $23: 13: 12.2$ & $-42: 24: 36$ & 0.05562 & 99 & - & Shectman et al. (1996) \\
\hline $23: 13: 13.3$ & $-42: 41: 07$ & 0.05495 & 0 & 15.013 & - \\
\hline $23: 13: 13.5$ & $-42: 27: 27$ & 0.05626 & 0 & 16.360 & - \\
\hline $23: 13: 16.0$ & $-42: 38: 11$ & 0.05673 & 0 & 16.195 & - \\
\hline $23: 13: 17.4$ & $-42: 22: 22$ & 0.05556 & 99 & - & Shectman et al. (1996) \\
\hline $23: 13: 25.5$ & $-42: 30: 32$ & 0.05432 & 1 & 16.204 & - \\
\hline $23: 13: 29.7$ & $-42: 28: 27$ & 0.05792 & 0 & 15.819 & Jones et al. (2009) \\
\hline $23: 13: 34.6$ & $-42: 38: 17$ & 0.05421 & 0 & 18.938 & - \\
\hline $23: 13: 37.5$ & $-42: 39: 42$ & 0.05716 & 0 & 20.064 & - \\
\hline $23: 13: 38.9$ & $-43: 01: 09$ & 0.05934 & 0 & 17.177 & - \\
\hline $23: 13: 41.4$ & $-42: 35: 20$ & 0.05424 & 0 & 19.584 & - \\
\hline $23: 13: 46.0$ & $-42: 45: 22$ & 0.05165 & 0 & 17.429 & - \\
\hline $23: 13: 48.8$ & $-42: 46: 34$ & 0.05246 & 0 & 15.274 & - \\
\hline $23: 13: 48.9$ & $-42: 47: 09$ & 0.05404 & 0 & 15.822 & - \\
\hline $23: 13: 49.6$ & $-42: 44: 44$ & 0.05583 & 0 & 17.653 & - \\
\hline $23: 13: 50.9$ & $-42: 45: 46$ & 0.05719 & 0 & 16.504 & - \\
\hline $23: 13: 52.3$ & $-42: 42: 39$ & 0.05817 & 0 & 19.396 & - \\
\hline $23: 13: 53.1$ & $-42: 36: 15$ & 0.05775 & $\theta$ & 15.123 & - \\
\hline $23: 13: 54.6$ & $-42: 47: 53$ & 0.05768 & 1 & 19.654 & - \\
\hline $23: 13: 54.7$ & $-42: 41: 18$ & 0.06083 & 0 & 17.371 & - \\
\hline $23: 13: 54.9$ & $-42: 43: 57$ & 0.05672 & 0 & 18.315 & - \\
\hline $23: 13: 57.2$ & $-42: 41: 39$ & 0.05463 & 0 & 15.999 & - \\
\hline $23: 13: 58.0$ & $-42: 37: 11$ & 0.05833 & 0 & 19.949 & - \\
\hline $23: 13: 58.6$ & $-42: 43: 39$ & 0.05651 & 0 & 14.537 & - \\
\hline $23: 14: 00.3$ & $-42: 52: 07$ & 0.05602 & 0 & 18.986 & - \\
\hline $23: 14: 01.3$ & $-42: 44: 26$ & 0.06196 & 0 & 16.000 & - \\
\hline $23: 14: 03.4$ & $-42: 29: 47$ & 0.05696 & 0 & 17.321 & - \\
\hline $23: 14: 03.5$ & $-42: 43: 11$ & 0.05778 & 0 & 17.559 & - \\
\hline $23: 14: 04.3$ & $-42: 38: 05$ & 0.05330 & 1 & 16.823 & - \\
\hline $23: 14: 04.3$ & $-42: 38: 24$ & 0.05737 & 0 & 17.827 & - \\
\hline $23: 14: 11.3$ & $-42: 36: 40$ & 0.05365 & 1 & 20.911 & - \\
\hline $23: 14: 16.5$ & $-42: 43: 16$ & 0.05499 & $\theta$ & 17.490 & - \\
\hline $23: 14: 21.0$ & $-42: 45: 01$ & 0.05713 & $\theta$ & 17.205 & - \\
\hline $23: 14: 22.1$ & $-42: 35: 10$ & 0.05266 & 0 & - & - \\
\hline $23: 14: 23.0$ & $-42: 39: 01$ & 0.05449 & 0 & 17.238 & - \\
\hline $23: 14: 25.5$ & $-42: 40: 47$ & 0.05693 & 0 & 15.714 & - \\
\hline $23: 14: 25.6$ & $-42: 33: 27$ & 0.05558 & 1 & 17.465 & - \\
\hline $23: 14: 26.9$ & $-42: 42: 28$ & 0.05604 & 0 & 16.157 & - \\
\hline $23: 14: 33.6$ & $-42: 20: 30$ & 0.05402 & $\theta$ & 17.025 & Shectman et al. (1996) \\
\hline $23: 14: 36.8$ & $-42: 42: 51$ & 0.05890 & 0 & 17.785 & - \\
\hline $23: 14: 49.9$ & $-42: 44: 24$ & 0.06118 & 1 & 19.876 & - \\
\hline $23: 14: 59.5$ & $-42: 47: 46$ & 0.05666 & 0 & 19.153 & - \\
\hline $23: 15: 00.9$ & $-42: 59: 54$ & 0.05667 & 1 & 17.837 & - \\
\hline $23: 15: 14.7$ & $-42: 07: 40$ & 0.05430 & 99 & - & Shectman et al. (1996) \\
\hline $23: 15: 30.9$ & $-42: 29: 14$ & 0.05486 & 99 & 16.274 & Zabludoff \& Mulchaey (1998) \\
\hline $23: 15: 52.0$ & $-42: 21: 24$ & 0.05396 & 1 & 16.590 & - \\
\hline $23: 16: 31.5$ & $-42: 23: 33$ & 0.05757 & 99 & - & Shectman et al. (1996) \\
\hline $23: 17: 02.3$ & $-42: 24: 41$ & 0.05349 & 0 & - & Shectman et al. (1996) \\
\hline
\end{tabular}

Notes. Columns 1, 2 show object positions based on GSC2.3 astrometry. The spectroscopic redshift is given in Col. 3. Column 4 refers to the spectral type: $\theta$ for passive spectra without emission lines, 1 for spectra with undoubtful [OII] or [OIII] lines, and 99 where poor data quality or the absence of spectra hampered classification. We assess a global uncertainty of our redshift measurements of $\Delta z \sim 0.00007$ (or $v \sim 22 \mathrm{~km} \mathrm{~s}{ }^{-1}$, see Sect. 3.1). We note that 376 out of the 403 individual sources with spectroscopic redshifts are within the field of our VIMOS pre-imaging. Column 5 gives the $R$-band magnitude in the Vega system for those sources from the VIMOS pre-imaging. Column 6 lists the references of the NED provided redshifts. 


\section{Appendix C: Properties of the combined spectroscopic sample}

We summarize the properties of all the VIMOS sources in our study below so that the community can access them either for cross-check or other purposes, for example identification of distant objects.

Table C.1. Properties of the combined spectroscopic sample (including NED entries).

\begin{tabular}{ccccc}
\hline \hline RA & Dec & $z$ & $R$ & Type \\
\hline $23: 12: 02.4$ & $-42: 58: 58$ & 0 & 20.675 & 2 \\
$23: 12: 02.9$ & $-42: 57: 22$ & 0 & 18.670 & 2 \\
$23: 12: 04.2$ & $-42: 46: 22$ & 0 & 21.063 & 2 \\
$23: 12: 40.6$ & $-42: 57: 27$ & 0 & 20.181 & 2 \\
$23: 12: 48.6$ & $-42: 46: 03$ & 0 & 13.557 & 2 \\
$23: 12: 50.7$ & $-42: 53: 48$ & 0 & 17.568 & 2 \\
$23: 12: 52.2$ & $-42: 55: 29$ & 0 & 20.256 & 2 \\
$23: 12: 55.5$ & $-42: 32: 02$ & 0 & 21.364 & 2 \\
$23: 12: 55.7$ & $-42: 41: 38$ & 0 & 20.559 & 2 \\
$23: 13: 18.2$ & $-42: 47: 20$ & 0 & 20.125 & 2 \\
$23: 13: 31.2$ & $-42: 35: 55$ & 0 & 20.672 & 2 \\
$23: 13: 31.6$ & $-42: 51: 50$ & 0 & 18.420 & 2 \\
$23: 13: 32.4$ & $-42: 32: 50$ & 0 & 20.263 & 2 \\
$23: 13: 37.4$ & $-42: 26: 55$ & 0 & 21.731 & 2 \\
$23: 13: 41.7$ & $-42: 34: 23$ & 0 & 14.277 & 2 \\
$23: 13: 53.6$ & $-42: 39: 34$ & 0 & 22.019 & 2 \\
$23: 13: 57.1$ & $-42: 56: 05$ & 0 & 15.085 & 2 \\
$23: 14: 09.8$ & $-42: 33: 26$ & 0 & 18.432 & 2 \\
$23: 14: 16.9$ & $-42: 53: 21$ & 0 & 20.796 & 2 \\
$23: 14: 19.6$ & $-42: 51: 26$ & 0 & 20.626 & 2 \\
$23: 14: 23.0$ & $-42: 43: 08$ & 0 & 20.275 & 2 \\
$23: 14: 25.8$ & $-42: 37: 18$ & 0 & 17.694 & 2 \\
$23: 14: 30.1$ & $-42: 52: 05$ & 0 & 20.844 & 2 \\
$23: 14: 32.8$ & $-42: 57: 58$ & 0 & 21.264 & 2 \\
$23: 14: 32.8$ & $-42: 57: 58$ & 0 & 21.264 & 2 \\
$23: 14: 36.3$ & $-42: 39: 18$ & 0 & 19.154 & 2 \\
$23: 14: 39.3$ & $-42: 43: 26$ & 0 & 19.205 & 2 \\
$23: 14: 42.0$ & $-42: 29: 14$ & 0 & 20.507 & 2 \\
$23: 14: 45.0$ & $-42: 33: 41$ & 0 & 16.645 & 2 \\
$23: 14: 50.4$ & $-42: 48: 57$ & 0 & 19.635 & 2 \\
$23: 15: 00.9$ & $-42: 59: 45$ & 0 & 18.288 & 2 \\
$23: 15: 05.7$ & $-42: 57: 27$ & 0 & 18.919 & 2 \\
$23: 15: 06.6$ & $-42: 34: 21$ & 0 & 20.943 & 2 \\
$23: 15: 10.5$ & $-42: 53: 24$ & 0 & 15.840 & 2 \\
$23: 15: 17.8$ & $-42: 59: 11$ & 0 & 21.111 & 2 \\
$23: 15: 22.0$ & $-42: 59: 25$ & 0 & 21.490 & 2 \\
$23: 15: 37.1$ & $-42: 23: 47$ & 0 & 20.301 & 2 \\
$23: 15: 38.2$ & $-42: 21: 50$ & 0 & 21.576 & 2 \\
$23: 15: 49.1$ & $-42: 24: 15$ & 0 & 20.795 & 2 \\
$23: 15: 52.2$ & $-42: 30: 56$ & 0 & 20.958 & 2 \\
$23: 15: 53.3$ & $-42: 29: 01$ & 0 & 21.066 & 2 \\
$23: 14: 57.3$ & $-42: 33: 30$ & 0.0060 & 15.930 & 1 \\
$23: 15: 33.1$ & $-42: 24: 40$ & 0.0282 & 20.073 & 0 \\
$23: 15: 07.1$ & $-42: 45: 59$ & 0.0416 & 18.355 & 1 \\
$23: 13: 46.0$ & $-42: 45: 22$ & 0.0516 & 17.429 & 0 \\
\hline & & & &
\end{tabular}

Notes. Among 403 objects in total, 376 found their matches in the source catalogue of the VIMOS pre-imaging. We list below their celestial positions (Cols. 1, 2), spectroscopic redshifts (Col. 3), $R$-band magnitude in the Vega system from the VIMOS pre-imaging (Col. 4), and spectral types (Col. 5). The spectral classification is based on the presence of emission lines in the spectrum; $\theta$ and 1 refer to passive and active galaxies, stars are indicated by 2 , and 99 indicates bad or missing spectra yielding no classification.
Table C.1. continued.

\begin{tabular}{|c|c|c|c|c|}
\hline RA & Dec & $z$ & $R$ & Type \\
\hline $23: 13: 48.8$ & $-42: 46: 34$ & 0.0525 & 15.274 & 0 \\
\hline $23: 14: 04.3$ & $-42: 38: 05$ & 0.0533 & 16.823 & 1 \\
\hline $23: 12: 15.2$ & $-42: 44: 28$ & 0.0535 & 17.611 & 0 \\
\hline $23: 14: 11.3$ & $-42: 36: 40$ & 0.0537 & 20.911 & 1 \\
\hline $23: 13: 48.9$ & $-42: 47: 09$ & 0.0540 & 15.822 & 0 \\
\hline $23: 14: 33.6$ & $-42: 20: 30$ & 0.0540 & 17.025 & 0 \\
\hline $23: 15: 52.0$ & $-42: 21: 24$ & 0.0540 & 16.590 & 1 \\
\hline $23: 13: 34.6$ & $-42: 38: 17$ & 0.0542 & 18.938 & 0 \\
\hline $23: 13: 41.4$ & $-42: 35: 20$ & 0.0542 & 19.584 & 0 \\
\hline $23: 13: 25.5$ & $-42: 30: 32$ & 0.0543 & 16.204 & 1 \\
\hline $23: 14: 23.0$ & $-42: 39: 01$ & 0.0545 & 17.238 & 0 \\
\hline $23: 13: 57.2$ & $-42: 41: 39$ & 0.0546 & 15.999 & 0 \\
\hline $23: 15: 30.9$ & $-42: 29: 14$ & 0.0549 & 16.274 & 99 \\
\hline $23: 13: 13.3$ & $-42: 41: 07$ & 0.0550 & 15.013 & 0 \\
\hline $23: 14: 16.5$ & $-42: 43: 16$ & 0.0550 & 17.490 & 0 \\
\hline $23: 12: 03.2$ & $-42: 54: 13$ & 0.0553 & 15.860 & 1 \\
\hline $23: 14: 25.6$ & $-42: 33: 27$ & 0.0556 & 17.465 & 1 \\
\hline $23: 13: 49.6$ & $-42: 44: 44$ & 0.0558 & 17.653 & 0 \\
\hline $23: 12: 53.1$ & $-42: 29: 19$ & 0.0559 & 18.837 & 1 \\
\hline $23: 13: 02.2$ & $-42: 36: 36$ & 0.0559 & 15.272 & 0 \\
\hline $23: 12: 40.5$ & $-42: 53: 16$ & 0.0560 & 16.208 & 0 \\
\hline $23: 12: 41.0$ & $-42: 54: 28$ & 0.0560 & 18.487 & 1 \\
\hline $23: 14: 00.3$ & $-42: 52: 07$ & 0.0560 & 18.986 & 0 \\
\hline $23: 14: 26.9$ & $-42: 42: 28$ & 0.0560 & 16.157 & 0 \\
\hline $23: 13: 13.5$ & $-42: 27: 27$ & 0.0563 & 16.360 & 0 \\
\hline 23:13:58.6 & $-42: 43: 39$ & 0.0565 & 14.537 & 0 \\
\hline $23: 13: 16.0$ & $-42: 38: 11$ & 0.0567 & 16.195 & 0 \\
\hline $23: 13: 54.9$ & $-42: 43: 57$ & 0.0567 & 18.315 & 0 \\
\hline $23: 14: 59.5$ & $-42: 47: 46$ & 0.0567 & 19.153 & 0 \\
\hline $23: 15: 00.9$ & $-42: 59: 54$ & 0.0567 & 17.837 & 1 \\
\hline $23: 14: 25.5$ & $-42: 40: 47$ & 0.0569 & 15.714 & 0 \\
\hline $23: 14: 03.4$ & $-42: 29: 47$ & 0.0570 & 17.321 & 0 \\
\hline $23: 14: 21.0$ & $-42: 45: 01$ & 0.0571 & 17.205 & 0 \\
\hline $23: 13: 06.0$ & $-42: 33: 21$ & 0.0572 & 18.481 & 0 \\
\hline $23: 13: 37.5$ & $-42: 39: 42$ & 0.0572 & 20.064 & 0 \\
\hline $23: 13: 50.9$ & $-42: 45: 46$ & 0.0572 & 16.504 & 0 \\
\hline $23: 14: 04.3$ & $-42: 38: 24$ & 0.0574 & 17.827 & 0 \\
\hline $23: 13: 53.1$ & $-42: 36: 15$ & 0.0577 & 15.123 & 0 \\
\hline $23: 13: 54.6$ & $-42: 47: 53$ & 0.0577 & 19.654 & 1 \\
\hline $23: 14: 03.5$ & $-42: 43: 11$ & 0.0578 & 17.559 & 0 \\
\hline $23: 13: 29.7$ & $-42: 28: 27$ & 0.0579 & 15.819 & 0 \\
\hline $23: 12: 19.9$ & $-42: 53: 50$ & 0.0580 & 15.518 & 0 \\
\hline $23: 13: 52.3$ & $-42: 42: 39$ & 0.0582 & 19.396 & 0 \\
\hline $23: 13: 58.0$ & $-42: 37: 11$ & 0.0583 & 19.949 & 0 \\
\hline $23: 14: 36.8$ & $-42: 42: 51$ & 0.0589 & 17.785 & 0 \\
\hline $23: 13: 38.9$ & $-43: 01: 09$ & 0.0593 & 17.177 & 0 \\
\hline $23: 13: 54.7$ & $-42: 41: 18$ & 0.0608 & 17.371 & 0 \\
\hline $23: 14: 49.9$ & $-42: 44: 24$ & 0.0612 & 19.876 & 1 \\
\hline $23: 14: 01.3$ & $-42: 44: 26$ & 0.0620 & 16.000 & 0 \\
\hline $23: 14: 00.1$ & $-42: 43: 02$ & 0.0632 & 18.313 & 0 \\
\hline $23: 14: 11.7$ & $-42: 50: 54$ & 0.0674 & 20.514 & 1 \\
\hline $23: 12: 55.8$ & $-42: 41: 47$ & 0.0675 & 19.938 & 1 \\
\hline $23: 14: 21.6$ & $-42: 45: 59$ & 0.0686 & 15.264 & 0 \\
\hline $23: 14: 43.4$ & $-42: 49: 53$ & 0.0686 & 18.405 & 1 \\
\hline $23: 15: 09.8$ & $-42: 56: 37$ & 0.0687 & 17.428 & 0 \\
\hline $23: 14: 18.9$ & $-42: 41: 04$ & 0.0689 & 18.306 & 1 \\
\hline $23: 12: 04.5$ & $-42: 44: 08$ & 0.0728 & 19.352 & 1 \\
\hline $23: 14: 12.3$ & $-42: 48: 49$ & 0.0880 & 17.456 & 0 \\
\hline $23: 13: 36.7$ & $-43: 00: 55$ & 0.0883 & 19.889 & 1 \\
\hline $23: 14: 39.2$ & $-42: 43: 28$ & 0.0883 & 18.021 & 1 \\
\hline
\end{tabular}


A. Rabitz et al.: Probing the dynamical and X-ray mass proxies of Abell S1101

Table C.1. continued.

\begin{tabular}{|c|c|c|c|c|}
\hline RA & Dec & $z$ & $R$ & Type \\
\hline $23: 14: 16.8$ & $-42: 49: 09$ & 0.0886 & 17.213 & 1 \\
\hline $23: 15: 06.1$ & $-42: 45: 42$ & 0.0886 & 20.193 & 1 \\
\hline $23: 14: 28.6$ & $-42: 56: 26$ & 0.0888 & 17.583 & 1 \\
\hline $23: 13: 29.4$ & $-42: 56: 41$ & 0.0889 & 20.435 & 1 \\
\hline $23: 13: 29.4$ & $-42: 56: 41$ & 0.0889 & 20.435 & 1 \\
\hline $23: 13: 12.3$ & $-42: 59: 02$ & 0.0890 & 19.318 & 1 \\
\hline $23: 13: 15.7$ & $-42: 44: 51$ & 0.0896 & 16.830 & 0 \\
\hline $23: 13: 13.6$ & $-42: 27: 31$ & 0.0914 & 16.360 & 1 \\
\hline 23:14:50.7 & $-42: 20: 06$ & 0.0914 & 18.269 & 1 \\
\hline $23: 13: 25.9$ & $-42: 58: 08$ & 0.0922 & 18.997 & 1 \\
\hline $23: 13: 27.5$ & $-42: 32: 05$ & 0.0983 & 17.569 & 1 \\
\hline 23:13:57.9 & $-42: 33: 02$ & 0.0985 & 19.212 & 0 \\
\hline 23:13:57.6 & $-42: 31: 44$ & 0.0986 & 16.170 & 0 \\
\hline 23:13:41.5 & $-42: 32: 42$ & 0.0992 & 21.087 & 0 \\
\hline 23:14:03.3 & $-42: 29: 13$ & 0.0992 & 16.414 & 1 \\
\hline $23: 13: 41.8$ & $-42: 34: 15$ & 0.0993 & 15.929 & 0 \\
\hline $23: 13: 49.5$ & $-42: 26: 34$ & 0.0993 & 20.185 & 1 \\
\hline 23:13:55.8 & $-42: 29: 51$ & 0.0993 & 19.445 & 1 \\
\hline $23: 14: 33.3$ & $-42: 39: 47$ & 0.0994 & 20.112 & 1 \\
\hline $23: 13: 46.9$ & $-42: 34: 38$ & 0.0995 & 15.879 & $\theta$ \\
\hline $23: 14: 13.1$ & $-42: 32: 23$ & 0.0995 & 18.922 & 1 \\
\hline $23: 13: 43.4$ & $-42: 28: 00$ & 0.0996 & 17.097 & 1 \\
\hline $23: 13: 55.4$ & $-42: 26: 19$ & 0.0996 & 19.460 & 1 \\
\hline 23:14:09.7 & $-42: 33: 33$ & 0.0996 & 17.321 & 1 \\
\hline $23: 12: 54.0$ & $-42: 30: 50$ & 0.0997 & 19.272 & 1 \\
\hline $23: 12: 51.7$ & $-42: 31: 05$ & 0.1000 & 18.019 & 0 \\
\hline $23: 13: 33.6$ & $-42: 27: 33$ & 0.1001 & 19.740 & 0 \\
\hline $23: 14: 07.7$ & $-42: 41: 17$ & 0.1001 & 17.723 & 1 \\
\hline $23: 13: 04.7$ & $-42: 52: 59$ & 0.1002 & 17.564 & 0 \\
\hline $23: 13: 12.0$ & $-42: 29: 34$ & 0.1002 & 15.940 & 0 \\
\hline $23: 13: 14.5$ & $-42: 29: 57$ & 0.1003 & 17.473 & 1 \\
\hline $23: 13: 52.7$ & $-42: 28: 47$ & 0.1003 & 16.793 & 0 \\
\hline $23: 15: 22.2$ & $-42: 34: 03$ & 0.1005 & 18.882 & 1 \\
\hline $23: 13: 52.8$ & $-42: 28: 23$ & 0.1006 & 16.729 & 0 \\
\hline $23: 13: 54.4$ & $-42: 29: 21$ & 0.1009 & 16.981 & 0 \\
\hline $23: 13: 14.3$ & $-42: 29: 59$ & 0.1012 & 17.473 & 1 \\
\hline $23: 15: 52.2$ & $-42: 25: 47$ & 0.1029 & 17.694 & 1 \\
\hline $23: 14: 25.7$ & $-42: 37: 28$ & 0.1039 & 18.984 & 0 \\
\hline $23: 13: 56.8$ & $-42: 39: 02$ & 0.1045 & 17.897 & 0 \\
\hline $23: 14: 28.0$ & $-42: 24: 49$ & 0.1046 & 20.361 & 1 \\
\hline $23: 14: 22.7$ & $-42: 38: 03$ & 0.1051 & 16.207 & 0 \\
\hline $23: 13: 50.8$ & $-42: 30: 00$ & 0.1055 & 19.993 & 1 \\
\hline $23: 13: 46.2$ & $-42: 30: 35$ & 0.1056 & 18.894 & 0 \\
\hline $23: 14: 20.5$ & $-42: 36: 21$ & 0.1057 & 18.423 & 1 \\
\hline $23: 13: 52.8$ & $-42: 28: 25$ & 0.1059 & 16.729 & 1 \\
\hline $23: 12: 17.8$ & $-42: 47: 10$ & 0.1065 & 18.340 & 0 \\
\hline $23: 13: 48.4$ & $-42: 31: 24$ & 0.1065 & 20.376 & 1 \\
\hline $23: 13: 48.4$ & $-42: 31: 24$ & 0.1065 & 20.376 & 1 \\
\hline $23: 13: 52.8$ & $-42: 28: 25$ & 0.1066 & 16.729 & 1 \\
\hline $23: 12: 13.8$ & $-42: 46: 36$ & 0.1067 & 16.777 & 0 \\
\hline $23: 12: 46.5$ & $-42: 56: 27$ & 0.1134 & 18.271 & 1 \\
\hline $23: 13: 57.2$ & $-42: 59: 11$ & 0.1149 & 19.788 & 1 \\
\hline $23: 14: 14.3$ & $-42: 46: 33$ & 0.1156 & 16.667 & 0 \\
\hline $23: 14: 59.8$ & $-42: 33: 44$ & 0.1156 & 19.156 & 1 \\
\hline $23: 15: 06.7$ & $-42: 35: 18$ & 0.1157 & 19.663 & 1 \\
\hline $23: 15: 11.5$ & $-42: 34: 52$ & 0.1158 & 17.540 & 1 \\
\hline $23: 13: 41.2$ & $-42: 42: 03$ & 0.1160 & 19.935 & 1 \\
\hline $23: 14: 28.1$ & $-42: 40: 16$ & 0.1163 & 17.790 & 0 \\
\hline 23:13:39.1 & $-42: 48: 23$ & 0.1164 & 18.580 & 1 \\
\hline $23: 12: 41.7$ & $-42: 28: 04$ & 0.1166 & 20.365 & 1 \\
\hline 23:14:30.9 & $-42: 42: 17$ & 0.1166 & 16.399 & 0 \\
\hline
\end{tabular}

Table C.1. continued.

\begin{tabular}{|c|c|c|c|c|}
\hline RA & Dec & $z$ & $R$ & Type \\
\hline $23: 13: 24.0$ & $-42: 53: 05$ & 0.1167 & 18.490 & $\theta$ \\
\hline 23:13:19.9 & $-42: 41: 49$ & 0.1182 & 21.422 & 1 \\
\hline $23: 13: 23.3$ & $-42: 51: 21$ & 0.1182 & 17.247 & 0 \\
\hline 23:13:07.2 & $-42: 56: 00$ & 0.1186 & 18.998 & 1 \\
\hline $23: 14: 23.0$ & $-42: 58: 28$ & 0.1208 & 17.618 & 1 \\
\hline 23:15:08.7 & $-42: 45: 23$ & 0.1244 & 20.195 & 1 \\
\hline $23: 14: 16.8$ & $-42: 53: 04$ & 0.1248 & 17.663 & 0 \\
\hline 23:13:34.2 & $-42: 54: 55$ & 0.1255 & 19.359 & 1 \\
\hline $23: 14: 42.9$ & $-42: 50: 25$ & 0.1258 & 17.884 & 0 \\
\hline $23: 14: 27.5$ & $-42: 56: 11$ & 0.1261 & 20.826 & 1 \\
\hline $23: 13: 31.3$ & $-42: 53: 19$ & 0.1327 & 18.859 & 1 \\
\hline $23: 14: 21.6$ & $-42: 56: 41$ & 0.1327 & 20.942 & 1 \\
\hline $23: 14: 25.9$ & $-42: 57: 26$ & 0.1327 & 18.149 & 1 \\
\hline 23:13:55.4 & $-42: 57: 46$ & 0.1338 & 21.261 & 1 \\
\hline $23: 12: 55.8$ & $-42: 30: 29$ & 0.1358 & 20.306 & 1 \\
\hline $23: 13: 10.6$ & $-42: 42: 59$ & 0.1410 & 20.071 & 1 \\
\hline $23: 12: 55.0$ & $-42: 37: 28$ & 0.1444 & 18.583 & 1 \\
\hline $23: 13: 52.3$ & $-42: 47: 34$ & 0.1445 & 20.025 & 1 \\
\hline 23:13:50.6 & $-42: 52: 02$ & 0.1449 & 17.232 & 1 \\
\hline $23: 14: 28.4$ & $-42: 53: 49$ & 0.1456 & 20.449 & 1 \\
\hline 23:15:00.4 & $-42: 52: 39$ & 0.1509 & 17.240 & 0 \\
\hline 23:15:06.7 & $-42: 52: 06$ & 0.1509 & 19.415 & 0 \\
\hline $23: 12: 02.5$ & $-42: 58: 29$ & 0.1526 & 17.943 & 1 \\
\hline $23: 14: 25.6$ & $-42: 23: 47$ & 0.1533 & 19.055 & 1 \\
\hline $23: 14: 56.8$ & $-42: 23: 27$ & 0.1534 & 18.630 & 0 \\
\hline 23:13:50.3 & $-42: 49: 56$ & 0.1540 & 17.647 & 0 \\
\hline 23:13:49.6 & $-42: 36: 58$ & 0.1542 & 18.016 & 1 \\
\hline $23: 15: 37.7$ & $-42: 34: 38$ & 0.1542 & 18.217 & 1 \\
\hline $23: 14: 30.6$ & $-42: 41: 34$ & 0.1543 & 18.686 & 1 \\
\hline $23: 14: 37.6$ & $-42: 21: 12$ & 0.1549 & 19.802 & 1 \\
\hline $23: 12: 43.5$ & $-42: 57: 07$ & 0.1550 & 17.469 & 0 \\
\hline 23:13:09.1 & $-42: 58: 19$ & 0.1591 & 18.067 & 1 \\
\hline 23:14:48.1 & $-42: 21: 46$ & 0.1617 & 19.329 & 0 \\
\hline $23: 14: 20.5$ & $-42: 53: 46$ & 0.1661 & 19.186 & 1 \\
\hline 23:11:45.1 & $-42: 48: 10$ & 0.1662 & 19.509 & 0 \\
\hline $23: 15: 40.3$ & $-42: 22: 12$ & 0.1679 & 20.249 & 1 \\
\hline 23:14:21.6 & $-42: 46: 56$ & 0.1698 & 18.845 & 1 \\
\hline 23:13:11.0 & $-42: 26: 55$ & 0.1700 & 20.542 & 1 \\
\hline 23:12:39.0 & $-42: 32: 28$ & 0.1706 & 18.304 & 1 \\
\hline 23:14:01.1 & $-42: 54: 33$ & 0.1709 & 19.810 & 1 \\
\hline $23: 15: 53.3$ & $-42: 23: 23$ & 0.1715 & 18.663 & 0 \\
\hline $23: 15: 34.8$ & $-43: 01: 35$ & 0.1716 & 18.682 & 1 \\
\hline $23: 13: 52.1$ & $-42: 35: 30$ & 0.1719 & 19.402 & 1 \\
\hline 23:14:06.0 & $-42: 55: 22$ & 0.1721 & 20.917 & 0 \\
\hline $23: 13: 46.7$ & $-42: 52: 35$ & 0.1724 & 18.143 & 1 \\
\hline 23:13:47.4 & $-42: 40: 02$ & 0.1725 & 20.430 & 1 \\
\hline 23:13:57.5 & $-42: 56: 14$ & 0.1726 & 18.615 & $\theta$ \\
\hline 23:12:55.8 & $-42: 37: 08$ & 0.1727 & 20.683 & 1 \\
\hline 23:13:28.6 & $-42: 55: 53$ & 0.1727 & 19.195 & 1 \\
\hline $23: 12: 48.6$ & $-42: 56: 41$ & 0.1728 & 19.207 & 1 \\
\hline 23:14:07.5 & $-43: 00: 10$ & 0.1729 & 19.857 & $\theta$ \\
\hline $23: 12: 49.6$ & $-42: 36: 15$ & 0.1732 & 19.187 & 1 \\
\hline 23:14:08.6 & $-43: 01: 41$ & 0.1738 & 19.125 & $\theta$ \\
\hline $23: 11: 48.5$ & $-42: 56: 27$ & 0.1754 & 18.220 & $\theta$ \\
\hline 23:15:00.3 & $-42: 37: 29$ & 0.1755 & 18.618 & 1 \\
\hline 23:12:06.8 & $-42: 55: 57$ & 0.1766 & 19.274 & 1 \\
\hline $23: 14: 55.5$ & $-42: 32: 49$ & 0.1793 & 19.472 & 0 \\
\hline 23:14:56.6 & $-42: 32: 22$ & 0.1799 & 19.632 & 1 \\
\hline $23: 14: 36.6$ & $-42: 48: 26$ & 0.1829 & 18.616 & 1 \\
\hline 23:13:31.9 & $-42: 53: 58$ & 0.1958 & 19.157 & 1 \\
\hline $23: 13: 58.3$ & $-42: 51: 33$ & 0.1963 & 18.246 & 1 \\
\hline
\end{tabular}


Table C.1. continued.

\begin{tabular}{|c|c|c|c|c|}
\hline RA & Dec & $z$ & $R$ & Type \\
\hline 23:14:09.7 & $-42: 47: 45$ & 0.1968 & 19.010 & 1 \\
\hline 23:14:26.1 & $-42: 41: 52$ & 0.2025 & 17.761 & 0 \\
\hline $23: 14: 39.0$ & $-42: 59: 01$ & 0.2026 & 19.262 & 0 \\
\hline $23: 14: 28.2$ & $-42: 59: 18$ & 0.2032 & 18.792 & 0 \\
\hline 23:14:40.1 & $-43: 02: 56$ & 0.2032 & 20.802 & 1 \\
\hline 23:14:13.6 & $-42: 54: 56$ & 0.2039 & 19.435 & 1 \\
\hline $23: 13: 52.9$ & $-43: 01: 51$ & 0.2040 & 19.318 & 1 \\
\hline 23:13:09.9 & $-42: 48: 02$ & 0.2062 & 19.662 & 1 \\
\hline $23: 14: 32.9$ & $-42: 47: 26$ & 0.2064 & 18.632 & 0 \\
\hline $23: 14: 33.3$ & $-42: 47: 36$ & 0.2067 & 19.422 & 0 \\
\hline $23: 14: 32.3$ & $-42: 47: 54$ & 0.2069 & 19.741 & 1 \\
\hline $23: 14: 14.7$ & $-42: 49: 42$ & 0.2072 & 18.716 & 1 \\
\hline $23: 15: 10.6$ & $-42: 49: 22$ & 0.2106 & 18.621 & 0 \\
\hline 23:14:39.7 & $-42: 50: 30$ & 0.2115 & 18.986 & 1 \\
\hline $23: 14: 59.9$ & $-42: 53: 55$ & 0.2150 & 19.220 & 0 \\
\hline 23:14:42.1 & $-42: 47: 55$ & 0.2155 & 17.756 & 0 \\
\hline 23:15:00.9 & $-42: 53: 06$ & 0.2159 & 18.463 & 0 \\
\hline 23:13:48.1 & $-42: 40: 34$ & 0.2164 & 19.909 & 1 \\
\hline $23: 13: 54.5$ & $-42: 38: 54$ & 0.2193 & 18.803 & 1 \\
\hline 23:14:06.6 & $-42: 37: 31$ & 0.2203 & 19.665 & 1 \\
\hline $23: 12: 19.2$ & $-42: 55: 39$ & 0.2239 & 20.155 & 1 \\
\hline $23: 11: 47.5$ & $-42: 56: 50$ & 0.2241 & 19.400 & 1 \\
\hline $23: 15: 31.7$ & $-43: 01: 52$ & 0.2254 & 18.682 & 0 \\
\hline 23:13:52.0 & $-42: 58: 35$ & 0.2300 & 20.465 & 1 \\
\hline $23: 11: 54.3$ & $-42: 45: 44$ & 0.2319 & 17.860 & 0 \\
\hline $23: 12: 43.5$ & $-42: 54: 46$ & 0.2319 & 18.493 & 0 \\
\hline $23: 13: 27.0$ & $-42: 49: 22$ & 0.2321 & 18.509 & 0 \\
\hline 23:13:24.1 & $-43: 00: 03$ & 0.2327 & 19.717 & 1 \\
\hline $23: 12: 50.8$ & $-42: 53: 40$ & 0.2341 & 19.433 & 1 \\
\hline $23: 14: 50.8$ & $-43: 00: 49$ & 0.2347 & 21.008 & 1 \\
\hline $23: 15: 26.0$ & $-42: 58: 33$ & 0.2356 & 19.157 & 1 \\
\hline $23: 14: 28.4$ & $-43: 02: 36$ & 0.2357 & 20.330 & 1 \\
\hline $23: 14: 35.2$ & $-43: 02: 11$ & 0.2358 & 20.499 & 1 \\
\hline $23: 15: 34.2$ & $-42: 48: 53$ & 0.2382 & 20.890 & 1 \\
\hline 23:12:05.2 & $-42: 53: 59$ & 0.2452 & 19.695 & 0 \\
\hline $23: 13: 15.0$ & $-42: 42: 33$ & 0.2471 & 19.196 & 1 \\
\hline 23:11:53.9 & $-42: 55: 00$ & 0.2481 & 20.513 & 1 \\
\hline 23:15:00.3 & $-42: 42: 17$ & 0.2519 & 20.353 & 1 \\
\hline 23:14:43.1 & $-43: 02: 29$ & 0.2520 & 20.199 & 1 \\
\hline 23:15:02.9 & $-42: 36: 25$ & 0.2539 & 18.991 & 1 \\
\hline $23: 15: 32.0$ & $-43: 02: 24$ & 0.2546 & 19.683 & 1 \\
\hline $23: 12: 17.7$ & $-42: 49: 00$ & 0.2571 & 20.310 & 1 \\
\hline $23: 14: 26.0$ & $-42: 31: 31$ & 0.2598 & 15.860 & 1 \\
\hline 23:14:57.9 & $-42: 35: 55$ & 0.2598 & 18.825 & 0 \\
\hline 23:15:00.2 & $-42: 37: 18$ & 0.2603 & 20.911 & 1 \\
\hline $23: 14: 34.9$ & $-42: 25: 11$ & 0.2605 & 20.291 & 1 \\
\hline 23:13:56.5 & $-42: 32: 16$ & 0.2612 & 21.443 & 1 \\
\hline $23: 14: 57.3$ & $-42: 38: 58$ & 0.2613 & 19.478 & 1 \\
\hline 23:13:54.6 & $-42: 27: 37$ & 0.2614 & 20.947 & 1 \\
\hline $23: 13: 10.4$ & $-42: 57: 57$ & 0.2620 & 19.264 & 0 \\
\hline $23: 13: 23.5$ & $-42: 50: 47$ & 0.2630 & 20.389 & 1 \\
\hline $23: 14: 52.2$ & $-42: 51: 42$ & 0.2687 & 19.410 & 0 \\
\hline $23: 14: 31.7$ & $-43: 01: 20$ & 0.2742 & 19.791 & 1 \\
\hline 23:13:59.7 & $-42: 33: 24$ & 0.2776 & 19.795 & 1 \\
\hline 23:12:05.2 & $-42: 45: 15$ & 0.2879 & 19.027 & 0 \\
\hline 23:13:13.7 & $-42: 45: 49$ & 0.2879 & 20.797 & 1 \\
\hline 23:13:03.9 & $-42: 59: 26$ & 0.2881 & 20.503 & 0 \\
\hline $23: 13: 23.2$ & $-42: 43: 41$ & 0.2899 & 20.717 & 0 \\
\hline $23: 14: 45.7$ & $-42: 45: 03$ & 0.2904 & 21.152 & 0 \\
\hline 23:13:27.1 & $-42: 29: 05$ & 0.2964 & 20.484 & 0 \\
\hline 23:15:18.9 & $-42: 50: 48$ & 0.3076 & 20.854 & 1 \\
\hline
\end{tabular}

Table C.1. continued.

\begin{tabular}{|c|c|c|c|c|}
\hline RA & Dec & $z$ & $R$ & Type \\
\hline $23: 15: 36.5$ & $-42: 32: 07$ & 0.3096 & 20.141 & 1 \\
\hline $23: 12: 54.6$ & $-42: 35: 53$ & 0.3097 & 17.726 & 1 \\
\hline 23:15:44.1 & $-42: 31: 40$ & 0.3098 & 20.391 & 1 \\
\hline $23: 14: 25.0$ & $-43: 00: 24$ & 0.3099 & 20.259 & 1 \\
\hline 23:13:13.8 & $-42: 50: 36$ & 0.3110 & 20.712 & 1 \\
\hline $23: 13: 26.8$ & $-42: 37: 56$ & 0.3114 & 20.390 & 1 \\
\hline $23: 12: 59.4$ & $-42: 38: 40$ & 0.3124 & 20.459 & 1 \\
\hline $23: 15: 25.1$ & $-42: 22: 34$ & 0.3209 & 19.837 & 1 \\
\hline 23:14:19.3 & $-42: 52: 42$ & 0.3211 & 19.695 & 1 \\
\hline $23: 14: 57.4$ & $-42: 20: 34$ & 0.3218 & 21.297 & 1 \\
\hline $23: 15: 21.9$ & $-42: 29: 57$ & 0.3261 & 19.562 & $\theta$ \\
\hline 23:14:26.6 & $-42: 30: 10$ & 0.3267 & 20.302 & 1 \\
\hline 23:11:59.6 & $-42: 49: 34$ & 0.3311 & 19.380 & 1 \\
\hline $23: 14: 27.2$ & $-42: 35: 53$ & 0.3431 & 20.116 & 0 \\
\hline $23: 14: 33.4$ & $-42: 22: 02$ & 0.3434 & 21.012 & 0 \\
\hline $23: 14: 33.4$ & $-42: 22: 02$ & 0.3434 & 21.012 & $\theta$ \\
\hline $23: 14: 47.3$ & $-42: 35: 36$ & 0.3444 & 20.094 & 1 \\
\hline $23: 14: 36.3$ & $-42: 44: 35$ & 0.3477 & 20.238 & $\theta$ \\
\hline $23: 15: 16.8$ & $-42: 50: 20$ & 0.3477 & 20.209 & 1 \\
\hline $23: 14: 36.3$ & $-42: 44: 38$ & 0.3483 & 20.242 & $\theta$ \\
\hline $23: 14: 53.4$ & $-43: 02: 11$ & 0.3485 & 19.508 & 1 \\
\hline 23:13:46.5 & $-42: 40: 16$ & 0.3496 & 18.877 & 1 \\
\hline 23:13:31.9 & $-42: 54: 05$ & 0.3498 & 18.929 & $\theta$ \\
\hline $23: 12: 58.7$ & $-42: 39: 42$ & 0.3525 & 20.191 & 0 \\
\hline $23: 14: 26.2$ & $-42: 31: 06$ & 0.3529 & 19.051 & 0 \\
\hline 23:13:06.6 & $-42: 44: 03$ & 0.3531 & 20.252 & 1 \\
\hline $23: 14: 15.5$ & $-43: 00: 53$ & 0.3540 & 20.507 & $\theta$ \\
\hline $23: 14: 48.4$ & $-42: 46: 51$ & 0.3546 & 19.719 & 1 \\
\hline 23:14:09.8 & $-42: 49: 59$ & 0.3550 & 20.905 & 1 \\
\hline 23:11:58.8 & $-42: 47: 46$ & 0.3603 & 19.840 & 1 \\
\hline $23: 12: 59.3$ & $-42: 44: 10$ & 0.3633 & 21.117 & 1 \\
\hline $23: 14: 26.0$ & $-42: 31: 27$ & 0.3681 & 15.860 & 1 \\
\hline 23:14:35.1 & $-42: 34: 24$ & 0.3681 & 14.351 & 1 \\
\hline 23:13:14.0 & $-42: 45: 09$ & 0.3693 & 20.695 & 1 \\
\hline 23:15:17.8 & $-42: 59: 07$ & 0.3693 & 21.111 & 1 \\
\hline 23:13:09.9 & $-42: 45: 22$ & 0.3699 & 20.293 & $\theta$ \\
\hline $23: 14: 54.4$ & $-42: 46: 34$ & 0.3700 & 19.926 & 1 \\
\hline 23:15:05.3 & $-42: 41: 33$ & 0.3703 & 19.499 & 1 \\
\hline $23: 14: 25.4$ & $-42: 23: 41$ & 0.3709 & 17.718 & 1 \\
\hline 23:15:56.2 & $-42: 30: 13$ & 0.3715 & 21.794 & 1 \\
\hline 23:13:02.4 & $-42: 39: 02$ & 0.3836 & 19.714 & 1 \\
\hline 23:13:06.4 & $-42: 42: 18$ & 0.3839 & 20.450 & 1 \\
\hline $23: 15: 34.8$ & $-42: 22: 52$ & 0.3885 & 21.098 & 1 \\
\hline 23:15:47.0 & $-42: 29: 20$ & 0.3887 & 20.422 & 0 \\
\hline $23: 15: 28.0$ & $-42: 25: 10$ & 0.3897 & 19.648 & $\theta$ \\
\hline 23:15:18.6 & $-42: 58: 05$ & 0.3939 & 21.943 & 1 \\
\hline 23:15:14.6 & $-42: 48: 29$ & 0.4059 & 19.947 & 1 \\
\hline $23: 15: 14.6$ & $-42: 48: 25$ & 0.4060 & 19.947 & 1 \\
\hline $23: 15: 16.5$ & $-43: 03: 05$ & 0.4074 & 21.331 & 0 \\
\hline $23: 15: 32.9$ & $-42: 31: 21$ & 0.4103 & 20.725 & 1 \\
\hline $23: 14: 30.0$ & $-42: 29: 01$ & 0.4109 & 19.580 & 1 \\
\hline $23: 14: 52.9$ & $-42: 38: 05$ & 0.4140 & 21.190 & 1 \\
\hline $23: 15: 58.0$ & $-42: 33: 05$ & 0.4140 & 19.613 & $\theta$ \\
\hline $23: 14: 32.7$ & $-42: 58: 01$ & 0.4154 & 20.151 & 1 \\
\hline 23:15:08.2 & $-42: 57: 07$ & 0.4156 & 20.690 & 1 \\
\hline $23: 15: 29.7$ & $-43: 00: 20$ & 0.4200 & 20.058 & 1 \\
\hline $23: 14: 50.9$ & $-42: 50: 39$ & 0.4202 & 20.784 & 1 \\
\hline 23:14:43.4 & $-42: 59: 57$ & 0.4213 & 20.840 & $\theta$ \\
\hline $23: 14: 56.0$ & $-42: 47: 28$ & 0.4213 & 20.795 & 1 \\
\hline $23: 14: 18.4$ & $-43: 01: 41$ & 0.4267 & 20.660 & 1 \\
\hline $23: 12: 42.3$ & $-42: 40: 27$ & 0.4387 & 21.078 & 0 \\
\hline
\end{tabular}


A. Rabitz et al.: Probing the dynamical and X-ray mass proxies of Abell S1101

Table C.1. continued.

\begin{tabular}{ccccc}
\hline \hline RA & Dec & $z$ & $R$ & Type \\
\hline $23: 13: 23.4$ & $-42: 48: 42$ & 0.4402 & 21.961 & 1 \\
$23: 14: 20.8$ & $-42: 59: 45$ & 0.4428 & 19.950 & 0 \\
$23: 13: 40.1$ & $-42: 50: 28$ & 0.4448 & 20.298 & 0 \\
$23: 15: 34.8$ & $-42: 33: 30$ & 0.4457 & 20.220 & 1 \\
$23: 14: 52.0$ & $-42: 25: 43$ & 0.4497 & 21.585 & 1 \\
$23: 14: 47.7$ & $-42: 30: 43$ & 0.4590 & 20.990 & 1 \\
$23: 14: 08.4$ & $-42: 35: 55$ & 0.4630 & 21.650 & 1 \\
$23: 12: 10.0$ & $-42: 50: 09$ & 0.4655 & 20.694 & 1 \\
$23: 14: 40.1$ & $-42: 43: 56$ & 0.4725 & 21.152 & 1 \\
$23: 15: 02.8$ & $-42: 35: 23$ & 0.4769 & 20.301 & 0 \\
$23: 14: 40.4$ & $-42: 53: 12$ & 0.4779 & 20.478 & 1 \\
$23: 14: 47.5$ & $-42: 22: 50$ & 0.4790 & 20.507 & 1 \\
$23: 15: 23.6$ & $-42: 35: 20$ & 0.4799 & 20.294 & 1 \\
$23: 14: 43.9$ & $-42: 38: 17$ & 0.4800 & 20.558 & 0 \\
$23: 15: 38.6$ & $-42: 35: 08$ & 0.4859 & 21.017 & 1 \\
$23: 12: 16.3$ & $-42: 59: 35$ & 0.4909 & 20.797 & 1 \\
$23: 13: 48.6$ & $-42: 30: 57$ & 0.5318 & 20.682 & 0 \\
$23: 13: 24.6$ & $-42: 37: 28$ & 0.5337 & 21.521 & 1 \\
$23: 14: 18.4$ & $-43: 01: 44$ & 0.5363 & 20.660 & 1 \\
$23: 12: 05.4$ & $-42: 57: 50$ & 0.5540 & 20.619 & 1 \\
$23: 15: 00.8$ & $-42: 51: 43$ & 0.5631 & 21.102 & 0 \\
$23: 13: 48.0$ & $-42: 56: 55$ & 0.5735 & 20.718 & 1 \\
$23: 12: 58.1$ & $-42: 28: 48$ & 0.5788 & 20.618 & 1 \\
$23: 14: 40.2$ & $-42: 29: 44$ & 0.6130 & 21.816 & 0 \\
$23: 14: 00.9$ & $-42: 46: 01$ & 0.6278 & 21.765 & 1 \\
$23: 13: 16.2$ & $-42: 43: 26$ & 0.6715 & 20.965 & 1 \\
$23: 13: 35.8$ & $-42: 46: 29$ & 3.4000 & 20.961 & 1 \\
\hline & & & &
\end{tabular}

